\title{
Hybrid Fuzzy - Stochastic 1D Site Response Analysis accounting for Soil Uncertainties
}

\author{
Alessandro Tombari ${ }^{\mathrm{a}, *}$, Luciano Stefanini $\mathrm{i}^{\mathrm{b}, * *}$ \\ ${ }^{a}$ School of Environment and Technology, University of Brighton, Brighton, UK \\ ${ }^{b}$ School of Economics, University of Urbino Carlo Bo, Urbino, IT
}

\begin{abstract}
The analysis of the seismic site response is conventionally carried out by the study of the one-dimensional amplification of vertically propagating shear waves through a horizontal soil profile with equivalent-linear elastic properties. Site response analysis requires the specification of the input ground motion and the dynamic characterization of the soil deposit. Whilst the stochastic approach is commonly used to model seismic excitations, the use of probability density functions for describing the soil properties is consistent only when precise information based on a large amount of data from soil surveys are available. Conversely, a non-probabilistic approach based on fuzzy set theory would be more appropriate for dealing with uncertainties that are just expressed by vague, imprecise, qualitative, or incomplete information supplied by engineering judgment. In this paper, we address a hybrid fuzzy-stochastic 1D site response analysis approach: we consider probability models for the seismic input and fuzzy intervals for dealing with soil uncertainties; the problem boundary values are defined as convex normal fuzzy sets and described by means of membership functions. Zadeh's extension principle, in combination with an efficient implementation of the Differential Evolution Algorithm for global minimization and maximization, is used to perform fuzzy computations. Results are presented as fuzzy median
\end{abstract}

\footnotetext{
${ }^{*}$ Principal corresponding author

** Corresponding author

Email address: lucste@uniurb.it (Luciano Stefanini)

$U R L:$ a.tombari@brighton.ac.uk (Alessandro Tombari)
}

Preprint submitted to Journal of Mechanical Systems and Signal Processing April 26, 2019 
value of the largest peaks of the peak ground acceleration at the surface by considering four types of soil classified in accordance with the European seismic building code. Finally, elastic response spectra defined in terms of gradual functions are proposed in order to evaluate the influence of the soil uncertainties on the seismic response of structures.

Keywords: Fuzzy logic, Site Response, Stochastic ground motion, Soil uncertainty

$X X X M S C$ : XX-XX, XX-XX

\section{Introduction}

Site response analysis aims to predict the influence of the local site effects on the characteristics of the earthquake ground motion. This analysis evaluates the propagation of the seismic waves through the soil deposit caused by the vibrations of the bedrock immediately beneath it. Under specific conditions such as the site is laterally infinite, horizontally layered, the ground surface is free of stresses, and the soil is stiffer with the depth, seismic waves propagate in the vertical, i.e. normal to the layers, direction. In this context, the most widely technique for the study of the one-dimensional amplification of vertically propagating waves involves the solution of the dynamic wave equation in the frequency domain with equivalent linear elastic soil properties (see, e.g., [1]). The most well-known implementations are represented by the code SHAKE [2] or EERA [3] that compute the equivalent linear solution of the soil propagation problem by the iterative scheme proposed by Seed and Idriss [4] where the soil properties are adjusted until they are compatible with the computed level of strain. This procedure is well established and site response analyses are exhaustively performed in the engineering practice in order to meet successfully seismic building code compliance by considering the expected earthquake event for selected values of return period for ground motion exceedance. Converse to the traditional Monte Carlo Simulation that requires a selection of multiple input rock motions for obtaining a statistically stable estimate of the median 
target response spectrum, random vibration theory (RVT) can be applied in order to assess statistically estimates of the response overcoming the problem of selection of a large number of earthquake input motions (e.g. see Rathje and Ozbey [5], and Deng and Ostadan [6]). Therefore, the ground input motion can be represented by a stationary Gaussian process, fully defined by the knowledge of its power density spectral (PSD) function determined from a seismological source model. Nevertheless, owing to the complexity of its intrinsic structure, the soil characterization manifests various sources of uncertainties due to the soil spatial variability and to the dispersion of the soil parameters. A current perspective is treating the soil as a random material, i.e., certain properties such as density, elastic modulus, shear modulus, etc., are described by statistical quantities. Manolis [7], in his review of the state-of-the-art in stochastic soil dynamics, distinguished among random loadings, random material properties and random boundaries. Moreover, in the context of site seismic response, a few of authors (see, e.g., Andrade and Borja [8], Rathje et al. [9]) analyzed the seismic response of soil deposits taking into account site property variabilities assuming normal or lognormal distribution of the soil uncertainties. On the other hand, due to the geologic process, the natural spatial variability of the soil can be relevant with observed strong variation of the properties even over small distances; soil properties maps generated from few soil surveys, do not provide sufficient information about soil deposits and rock formation. The genuine lack of knowledge or imprecision in the definition of a property, in addition to the dispersion of the data caused by systematic measurement errors, fluctuations and sample disturbance, determine unavoidable uncertainty of an epistemic nature. Due to the large amount of data required to estimate the parameters for the dynamic geotechnical characterization of the soil deposit, the use of probability density functions for all of them becomes inconsistent; a probabilistic model dealing with these uncertainties requires supplying greater knowledge than that gained from actual experience. Oberguggenberger [10] showed, for geotechnical systems, the high sensitivity in calculating the failure probability when different distributions obtained by fitting the same input data from laboratory tests 
are used. Therefore, in order to avoid misleading representations, several approaches alternative to the probabilistic method, referred to as non-probabilistic methods, have been developed (see, e.g., Booker and Ross [11], Beck et al, [12, Beer et al. [13]). In particular, fuzzy set theory (Zadeh [14]) can be applied for dealing with non-random, incomplete, imprecise information as well as linguistic vagueness, namely the use of natural linguistic information in engineering judgment knowledge, for classifying generic class of soil (e.g. soft, medium, rigid) or the soil deposit type (e.g. class A-B-C-D according to the EN 1998-1, [15]). Fuzzy set theory (see, e.g., Hanss [16], Bede [17], Ross [18]) uses the concept of possibility in which a fuzzy set $\tilde{A}$, is described as a class of objects with a continuum of grades of membership $\mu_{\tilde{A}}$, ranging from $\alpha=0$ (the object does not belong to the set) to $\alpha=1$ (the object completely belongs to the set). In literature, several authors have had recourse to the fuzzy logic for dealing with uncertainties in soil properties; just to cite a few of them, Zhang and Tumay [19] introduced a fuzzy set for the Cone Penetration Test (CPT) soil engineering classification, Romo and Garcia [20] determined a Neuro-Fuzzy-Network mapping the experimental data of cone penetration tests into dynamic properties such as the shear wave velocity, 21] used a Fuzzy-Neural Network Method to describe uncertain input parameters for soil-structure interaction problems, and Valdebenito et al, 22] analyzed the vertical deflection of a cylindrical pile in elastic bi-layered soil by considering approximate fuzzy parameters for soil and pile properties. In seismic engineering, Marano et al. [23] applied the concept of credibility for determining a fuzzy concept of stochastic seismic response spectrum by applying the Clough and Penziens double filter [24], where the filter parameters were considered to be fuzzy. Clough and Penziens approach simplifies the response of the soil as linear single degree of freedom (SDOF) in which the natural frequency and the critical damping ratio are the only parameters required to describe the soil response.

However, for vulnerable site or critical structures, a site-specific seismic analysis, referred to as site response analysis, that reflects to the local site conditions is required. 
In this paper, the main parameters involved in the site response analysis, i.e. the shear wave modulus $G_{0}$, the soil unit density $\rho$, the maximum damping ratio $\xi_{f}$, and the depth of the soil deposit $h$ are considered by using the fuzzy logic approach for dealing with the unavoidable uncertainties which occur during soil characterization. On the other hand, seismic excitation defined at the outcrop bedrock is described through a stochastic approach (see e.g., 25]). The membership function of the fuzzy output $\tilde{a}_{P G A}\left(\tilde{h}, \tilde{G}_{0}, \tilde{\rho}, \tilde{\xi_{f}}\right)$ representing the fuzzy median value of the largest peaks of the free field acceleration at the top surface, as a function of the fuzzy parameters $\tilde{G}_{0}, \tilde{\rho}, \tilde{h}$ and $\tilde{\xi}$, is obtained by the Zadeh's extension principle, in combination with an efficient implementation of the Differential Evolution Algorithm for global minimization and maximization (Stefanini [26]).

Results are presented as fuzzy median value of the largest peaks of the peak ground acceleration at the ground surface by considering four types of soil classified in accordance with the European seismic building code (i.e. soil type A-B-C-D).

Moreover, by accounting for deterministic structural parameters, elastic response spectra described in terms of gradual functions, are evaluated in order to analyse the influence of the soil uncertainties on the seismic response of structures.

\section{Stochastic 1D Site Response Analysis Problem}

This section derives the stochastic equation of the one-dimensional (1D) site response analysis problem through the application of the random vibration theory when only the seismic event is considered uncertain, in particular random. The site response analysis aims to evaluate the effects of the local soil conditions on the amplitude and frequency content of the seismic motion that propagates through the soil deposit during an earthquake event. Figure 1 describes the problem investigated in this paper: the ground motion $U_{\text {out }}(\omega)$, where $\omega \geq 0$ is the circular frequency, is known at the bedrock outcrop where accelerometer 
stations are usually installed for recording real earthquake events; this random ground motion (Step 1) is modelled as a zero-mean stationary Gaussian stochastic process fully described by the knowledge of its power spectral density function, denoted by $S_{\ddot{U}_{0} \ddot{U}_{0}}(\omega)$. After that, the input ground motion process $U_{\text {out }}(\omega)$ is derived by transferring the ground motion from the bedrock outcrop to the bedrock underling the soil deposit, obtaining $S_{U_{g} U_{g}}(\omega)$ (Step 2). The site response analysis consists in predicting the stochastic ground motion process $U(\omega, z)$, where $z$ is the depth from the ground surface, propagating vertically through the soil deposit (Step 3), caused by the stochastic seismic motion process $U_{g}(\omega)=U\left(\omega, z_{\text {bed }}\right)$ at the bedrock level $\left(z=z_{\text {bed }}\right)$. Finally, the ground motion process at the soil deposit surface $U(\omega)=U(\omega, 0)$ is described by determining its power spectral density (Step 4) as well as the acceleration response spectrum (Step 5).

Under the assumption of horizontal soil layers of infinite extent, the study of shear waves propagating vertically is accomplished by solving the one-dimensional soil amplification problem described in the frequency domain by the following dynamic equation [1]:

$$
G^{*} \frac{d^{2} U(\omega, z)}{d z^{2}}=\rho \omega^{2} U(\omega, z)
$$

Here, $\rho$ is the soil density and $G^{*}$ is the complex linear equivalent shear modulus of the soil defined as:

$$
G^{*}=G(1+2 \mathbf{i} \xi)
$$

in which $G$ and $\xi$ are the real-valued secant shear modulus and critical damping ratio and $\mathbf{i}$ is the imaginary unit.

By considering a layered continuous soil deposit composed of $n$ layers of which each $i-t h$ layer is characterized by constant properties $G_{i}, \rho_{i}$ and $\xi_{i}$, and its own local coordinate system $\zeta_{i}$ as illustrated in Figure 1, the wave equation of the $i-t h$ layer assumes the form given in Eq. (1) that admits the following steady state general solution:

$$
U\left(\omega, \zeta_{i}\right)=U_{i}(\omega)=C_{i}(\omega) e^{\mathbf{i} k_{i}^{*} \zeta_{i}}+D_{i}(\omega) e^{-\mathbf{i} k_{i}^{*} \zeta_{i}}
$$




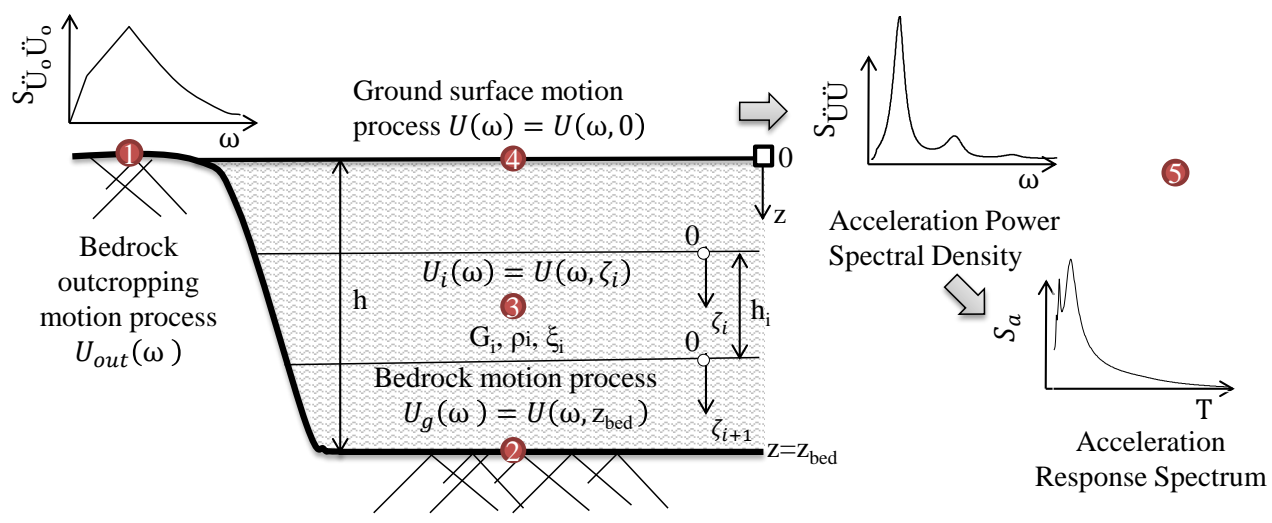

Figure 1: Stochastic 1D site response analysis procedure adopted in this paper

where $k_{i}^{*}=\sqrt{\frac{\rho_{i} \omega^{2}}{G_{i}^{*}}}$ is the complex wave number as a function of the complex shear modulus $G_{i}^{*}$ given by Eq. (2) for the $i-t h$ layer, and $C_{i}(\omega)$ and $D_{i}(\omega)$ are the amplitudes of waves travelling downwardly and upwardly, respectively.

Equation (1) is addressed for each layer by computing the transfer function between the $i-t h$ displacement $U_{i}(\omega)$, calculated at the top of the layer $\zeta_{i}=0$, and the excitation $U_{g}(\omega)$ applied at the bedrock, as follows:

$$
H_{i}(\omega)=\frac{U_{i}(\omega)}{U_{g}(\omega)}=\frac{C_{i}(\omega)+D_{i}(\omega)}{C_{g}(\omega)+D_{g}(\omega)}
$$

where $C_{i}(\omega)$ and $D_{i}(\omega)$, for $i=1, \ldots, n$ are the coefficients of Eq. (3) for the $i-t h$ layer characterized by constant equivalent linear properties and layer thickness $h_{i}$ whereas $C_{g}(\omega)$ and $D_{g}(\omega)$, are related to the bedrock properties. The coefficients are obtained by the recursion formula as coded in the computer program SHAKE [2] as follows (the dependence on $\omega$ has been omitted for notational simplicity):

$$
\begin{aligned}
& C_{i+1}=0.5 C_{i}\left(1+\beta_{i}\right) e^{\mathrm{i} k_{i} h_{i}}+0.5 D_{i}\left(1-\beta_{i}\right) e^{-\mathbf{i} k_{i} h_{i}} \\
& D_{i+1}=0.5 C_{i}\left(1-\beta_{i}\right) e^{\mathrm{i} k_{i} h_{i}}+0.5 D_{i}\left(1+\beta_{i}\right) e^{-\mathbf{i} k_{i} h_{i}}
\end{aligned}
$$

where $\beta_{i}=\frac{k_{i} G_{i}^{*}}{k_{i+1} G_{i+1}^{*}}$. In Eq. 44 , the stochastic ground motion process $U_{g}(\omega)$ is known through its power spectral density function $S_{U_{g} U_{g}}(\omega)$; therefore, by applying the basics of the random vibration theory, the power spectral density 
function $S_{U U, i}(\omega)$ of the displacement response at the $i-t h$ layer, subjected to the Gaussian stationary process $U_{g}(\omega)$, is computed as follows:

$$
S_{U U, i}(\omega)=\left|H_{i}(\omega)\right|^{2} S_{U_{g} U_{g}}(\omega)
$$

Moreover, from the definition of the shear strain $\gamma\left(\omega, \zeta_{i}\right)=\frac{d U\left(\omega, \zeta_{i}\right)}{d \zeta_{i}}$, the shear strain of the $i-t h$ layer is:

$$
\gamma_{i}(\omega)=\mathbf{i} k_{i}^{*}\left(C_{i}(\omega) e^{\mathbf{i} k_{i}^{*} \zeta_{i}}+D_{i}(\omega) e^{-\mathbf{i} k_{i}^{*} \zeta_{i}}\right)
$$

Therefore, the power spectral density of the shear strain $S_{\gamma \gamma, i}(\omega)$ is calculated as:

$$
S_{\gamma \gamma, i}(\omega)=\left|\gamma_{i}(\omega)\right|^{2} \frac{S_{U U, i}(\omega)}{2} .
$$

Statistical quantities of the response are thus derived from the stochastic Eqs. (7) and (9); in particular, the characteristic shear strain $X_{\gamma}$ and the characteristic acceleration $X_{U}$ at each iteration are computed as the fractile of order $p$ (usually the median, i.e. $p=0.5$ ) of the distribution of maxima through the first crossing problem [27] defined as follows:

$$
X_{\gamma, i}=\eta_{\gamma, i}\left(T_{s}, p\right) \sqrt{\lambda_{0, \gamma, i}}
$$

and

$$
X_{\ddot{U}, i}=\eta_{\ddot{U}, i}\left(T_{s}, p\right) \sqrt{\lambda_{0, \ddot{U}, i}} .
$$

Here, $\eta_{\gamma, i}$ and $\eta_{\ddot{U}, i}$ are peak factors determined through the relation obtained by [28] and adapted for the shear strain and acceleration respectively:

$$
\eta_{\gamma, i}=\sqrt{2 \ln \left(2 N_{\gamma}\left(1-e^{\left.-\delta_{\gamma, i}^{1,2} \sqrt{\pi \ln \left(2 N_{\gamma, i}\right)}\right)}\right)\right.}
$$

and

$$
\eta_{\ddot{U}, i}=\sqrt{2 \ln \left(2 N_{\ddot{U}}\left(1-e^{-\delta_{\ddot{U}, i}^{1.2} \sqrt{\pi \ln \left(2 N_{\ddot{U}, i}\right)}}\right)\right)}
$$

with

$$
N_{\gamma, i}=\frac{T s}{-2 \pi \ln (p)} \frac{\lambda_{2, \gamma, i}}{\lambda_{0, \gamma, i}}
$$

and

$$
N_{\ddot{U}, i}=\frac{T s}{-2 \pi \ln (p)} \frac{\lambda_{2, \ddot{U}, i}}{\lambda_{0, \ddot{U}, i}}
$$


as well as

$$
\delta_{\gamma, i}=\sqrt{1-\frac{\lambda_{1, \gamma, i}^{2}}{\lambda_{0, \gamma, i} \lambda_{2, \gamma, i}}}
$$

and

$$
\delta_{\ddot{U}, i}=\sqrt{1-\frac{\lambda_{1, \ddot{U}, i}^{2}}{\lambda_{0, \ddot{U}, i} \lambda_{2, \ddot{U}, i}}} ;
$$

in the previous relations, $T_{s}$ is the time length of the stationary part of the signal and the zeroth-order, first-order, second-order response spectral moments of the acceleration, $\lambda_{r, \ddot{U}, i}$, for $r=0,1$, and 2 , respectively, are expressed as

$$
\lambda_{r, \ddot{U}, i}=\int_{0}^{\infty} \omega^{4+r} S_{U U, i}(\omega) \mathrm{d} \omega,
$$

whereas the he zeroth-order, first-order, second-order response spectral moments of the shear strain $\lambda_{r, \gamma, i}$ for $r=0,1$, and 2 , respectively, are given by:

$$
\lambda_{r, \gamma, i}=\int_{0}^{\infty} \omega^{r} S_{\gamma \gamma, i}(\omega) \mathrm{d} \omega .
$$

The characteristic shear strain $X_{\gamma}$ is used for evaluating the secant soil properties through the iterative scheme proposed by 4 as equivalent linear properties; the secant modulus $G_{i}$ and the critical damping ratio $\xi_{i}$ are determined as follows:

$$
\begin{aligned}
& G_{i}=G_{0, i} f\left(\gamma_{i}^{r}\right) \\
& \xi_{i}=\xi_{f, i} g\left(\gamma_{i}^{r}\right)
\end{aligned}
$$

where $G_{0, i}$ and $\xi_{f, i}$ are the initial shear modulus and the maximum damping ratio, respectively whereas $f\left(\gamma_{i}^{r}\right)$ and $g\left(\gamma_{i}^{r}\right)$ are the modulus reduction and the damping ratio curves, respectively, calculated at the reference or characteristic shear strain $\gamma_{i}^{r}$ of the $i-t h$ layer, defined as the median $(p=0.5)$ characteristic strain of the stochastic process as follows:

$$
\gamma_{i}^{r}=R_{\gamma} \gamma_{i}^{\text {median }}=R_{\gamma} X_{\gamma}
$$

The term $R_{\gamma}$ of Eq. 21) is a coefficient accounting for the effect of the transient response on the material properties, usually assumed constant equal to 0.65 [1]

Generally, the seismic input at the bedrock level, $U_{g}(\omega)$, is derived from a given seismic load process $U_{\text {out }}(\omega)$ that is known at the rock outcrop where 
accelerometer stations are installed; the power spectral density at the bedrock, $S_{U_{g} U_{g}}(\omega)$, is evaluated from the following expression:

$$
S_{U_{g} U_{g}}(\omega)=\left|\frac{2 C_{g}(\omega)}{C_{g}(\omega)+D_{g}(\omega)}\right|^{2} S_{U_{0} U_{0}}(\omega) .
$$

Finally, in order to obtain the stochastic elastic response spectrum of Step 5 as depicted in Figure 1, the RVT is exploited to calculate the median value of largest peak of the response of each single linear oscillator (SDOF) system subjected to the ground motion process $S_{\ddot{U} \ddot{U}}(\omega)$. It is worth mentioning that in case of nonstationary processes, an equivalent stochastic linearisation approach can be used (see e.g, 29]). For each structural period $T$, the pseudo-acceleration elastic response spectrum may be directly obtained as follows:

$$
S_{a}(T)=\eta_{\ddot{U}} \sqrt{\lambda_{0, \ddot{U}}}
$$

In Eq. 23, $\eta_{\ddot{U}}$ and $\lambda_{0, \ddot{U}}$, i.e. the peak factor in Eq. 11 and the zeroth-order spectral moment of Eq. 18 respectively, are computed by using the transfer function of the single degree of freedom $H_{S D O F}$ at the period $T$, i.e. at $\omega_{0}=\frac{2 \pi}{T}$ :

$$
H_{S D O F}(\omega)=-\left(\frac{\omega_{0}^{2}}{\omega^{2}}+\mathbf{i} 2 \xi \frac{\omega_{0}}{\omega}-1\right)^{-1}
$$

in place of the the soil deposit transfer function in Eq. (7), whereas the input is power spectral density in term of acceleration $S_{\ddot{U} \ddot{U}}(\omega)$, derived from the relation $S_{\ddot{U} \ddot{U}}(\omega)=\omega^{4} S_{U_{0} U_{0}}(\omega)$, obtained by the soil propagation problem in Eq. (7). Therefore, the stochastic 1D site response analysis problem for an equivalent linear soil deposit is fully determined.

\section{Fuzzy Approach to Soil Parameter Uncertainty}

The stochastic 1D site response analysis when soil parameters are uncertain, is hereafter established by exploiting the fuzzy logic approach. In the proposed soil amplification problem, in combination with the random nature of the input motion, sources of uncertainty include variability in material properties, such as the shear elastic modulus, the density, and the damping ratio as well as 
geometric boundaries as the thickness of the soil deposit. These uncertainties are mainly caused by measurement errors, sampling disturbance and/or incomplete knowledge about soil description; moreover, the use of natural language for classifying the ground type (e.g. soft, soft-to-firm, stiff, very stiff) or the soil type (e.g. Unified Soil Classification System) is common among practitioners. In this context, Fuzzy sets theory (Zadeh [14]) has been shown to be effective for dealing with the epistemic nature of these uncertainties (e.g see Hanss and Turrin 30] and Fetz et al. 31]). Especially, when evidences do not allow a probability interpretation of the data sets, fuzzy logic is a reasonable approach for capturing the vagueness meaning of their properties; moreover, contrary to the use of interval analysis, where only upper and lower bounds are assigned to each parameter, the fuzzy sets provide further information about the grade of possibility (or possibility distribution) on the interval. This section gives a brief introduction on fuzzy logic interpretation of the investigated system parameters as well as on fuzzy algebra and strategies to solve the optimal fuzzy problem.

\subsection{Basic Fuzzy Theory}

Given a system parameter $A$, its representation as a fuzzy set $\tilde{A}$ over the set of real numbers $\mathbb{R}$ (the universe) is usually defined by its membership function

$$
\mu_{\tilde{A}}: \mathbb{R} \longrightarrow[0,1]
$$

and a fuzzy (sub)set $\tilde{A}$ of $\mathbb{R}$ is uniquely characterized by the pairs $\left(x, \mu_{\tilde{A}}(x)\right)$ for each $x \in \mathbb{R}$; the value $\mu_{\tilde{A}}(x) \in[0,1]$ is the membership grade of $x$ to the fuzzy set $\tilde{A}$. If $\mu_{\tilde{A}}$ assumes only the two values 0 or 1 , we obtain a subset of $\mathbb{R}$ in the classical set-theoretic sense (what is called a crisp set in the fuzzy context) and $\mu_{\tilde{A}}$ is simply the characteristic function of $\tilde{A}$. Fundamental concepts in fuzzy theory (see, e.g., [17]) are the support, the level-sets (or level-cuts) and the core of a fuzzy set (or of its membership function). The support of $\tilde{A}$ is the (crisp) subset of points of $x \in \mathbb{R}$ at which the membership grade $\mu_{\tilde{A}}(x)$ is positive:

$$
\operatorname{supp}(\tilde{A})=\left\{x \mid x \in \mathbb{R}, \mu_{\tilde{A}}(x)>0\right\}
$$


we always assume that $\operatorname{supp}(\tilde{A}) \neq \emptyset$. For $\alpha \in] 0,1]$, the $\alpha$-level cut of $\tilde{A}$ (or simply the $\alpha-$ cut) is defined by

$$
[\tilde{A}]_{\alpha}=\left\{x \mid x \in \mathbb{R}, \mu_{\tilde{A}}(x) \geq \alpha\right\}
$$

and for $\alpha=0$ by the closure of the support

$$
[\tilde{A}]_{0}=\operatorname{cl}\left\{x \mid x \in \mathbb{R}, \mu_{\tilde{A}}(x)>0\right\} .
$$

The core of $\tilde{A}$ is the set of elements of $\mathbb{R}$ having membership grade 1

$$
\operatorname{core}(\tilde{A})=\left\{x \mid x \in \mathbb{R}, \mu_{\tilde{A}}(x)=1\right\}
$$

and we say that $\tilde{A}$ is normal if $\operatorname{core}(\tilde{A}) \neq \emptyset$.

A particular class of fuzzy sets is when the level-cuts $[\tilde{A}]_{\alpha}$ are compact intervals or equivalently, the membership function is upper semi-continuous and quasi-concave. In this case, a fuzzy set $\tilde{A}$ is called a fuzzy number if $\exists \widehat{A} \in \mathbb{R}$ such that $\operatorname{core}(\tilde{A})=\{\widehat{A}\}$, and is called a fuzzy interval if $\exists \widehat{A}^{-}, \widehat{A}^{+} \in \mathbb{R}, \widehat{A}^{-}<\widehat{A}^{+}$ such that $\operatorname{core}(\tilde{A})=\left[\widehat{A}^{-}, \widehat{A}^{+}\right]$.

We will denote by $\mathcal{F}^{1}$ the space of real (unidimensional) fuzzy intervals with the above properties; the $\alpha$-cuts of a fuzzy number or interval are compact intervals of the form

$$
[\tilde{A}]_{\alpha}=\left[A_{\alpha}^{-}, A_{\alpha}^{+}\right] \subset \mathbb{R}
$$

If $A_{\alpha}^{-}=\widehat{A}^{-}$and $A_{\alpha}^{+}=\widehat{A}^{+}, \forall \alpha \in[0,1]$ we have a crisp interval or a crisp number (if, in addition, $\widehat{A}^{-}=\widehat{A}^{+}$). If $A_{\alpha}^{-}+A_{\alpha}^{+}=\widehat{A}^{+}+\widehat{A}^{-}, \forall \alpha \in[0,1]$ then the fuzzy interval is called symmetric. We say that $\tilde{A}$ is positive if $A_{\alpha}^{-}>0, \forall \alpha \in[0,1]$ and that $\tilde{A}$ is negative if $A_{\alpha}^{+}<0, \forall \alpha \in[0,1]$. Any fuzzy number or interval $\tilde{A} \in \mathcal{F}^{1}$ has the well known LR-representation (L for left, $\mathrm{R}$ for right), i.e. its membership function is of the form

$$
\mu_{\tilde{A}}(x)= \begin{cases}A^{L}(x) & \text { if } a \leq x<c \\ 1 & \text { if } c \leq x \leq d \\ A^{R}(x) & \text { if } d<x \leq b \\ 0 & \text { otherwise }\end{cases}
$$


where $a<c \leq d<b$, the function $A^{L}:[a, c] \rightarrow[0,1]$ is non-decreasing with $A^{L}(a)=0, A^{L}(c)=1$ and the function $A^{R}:[d, b] \rightarrow[0,1]$ is non-increasing with $A^{R}(d)=1, A^{R}(b)=0$. The interval $[a, b]$ is the support and $[c, d]$ is the core. If $c=d$, we obtain a fuzzy number. We refer to the functions $A^{L}($.$) and$ $A^{R}($.$) as the left and right sides of \tilde{A}$, respectively and we refer to the functions $A_{(.)}^{-}$and $A_{(.)}^{+}$as the lower and upper branches of $\tilde{A}$, respectively.

The simplest fuzzy intervals have linear branches (in the LR or LU representations). A trapezoidal fuzzy interval, denoted by $\tilde{A}=\langle a, c, d, b\rangle$, where $a \leq c \leq d \leq b$, has $\alpha-c u t s$

$$
[\tilde{A}]_{\alpha}=[a+\alpha(c-a), b-\alpha(b-d)], \alpha \in[0,1] .
$$

If $c=d$ we obtain a triangular fuzzy number, denoted by $\tilde{A}=\langle a, c, b\rangle$, where $a<c<b$ and the core is a singleton c. Given two fuzzy numbers $\tilde{A}, \tilde{B} \in \mathcal{F}^{1}$, the four arithmetic operations are defined by the use of Zadeh's extension principle $(\circ \in\{+,-, \times, /\}):$

$$
\mu_{\tilde{A} \circ \tilde{B}}(z)=\sup _{z=x \circ y} \min \left\{\mu_{\tilde{A}}(x), \mu_{\tilde{B}}(y)\right\} .
$$

More generally, we will denote by $\mathcal{F}^{n}$ the space of vectors $\tilde{\mathbf{A}}=\left(\tilde{A}_{1}, \tilde{A}_{2}, \ldots, \tilde{A}_{n}\right)$ with $n$ components $\tilde{A}_{j} \in \mathcal{F}^{1}$ for $j=1, \ldots, n$. Consider the extension of a function $f: \mathbb{R}^{n} \rightarrow \mathbb{R}$ to a vector $\tilde{\mathbf{A}}=\left(\tilde{A}_{1}, \tilde{A}_{2}, \ldots, \tilde{A}_{n}\right) \in \mathcal{F}^{n}$ of $n$ fuzzy numbers, with $k$-th component $\tilde{A}_{k} \in \mathcal{F}^{1}$ given, in terms of $\alpha$-cuts, by $\left[\tilde{A}_{k}\right]_{\alpha}=\left[A_{k, \alpha}^{-}, A_{k, \alpha}^{+}\right]$ for $k=1,2, \ldots, n$. Denote by $\tilde{B}=\tilde{f}\left(\tilde{A}_{1}, \tilde{A}_{2}, \ldots, \tilde{A}_{n}\right)$ the corresponding fuzzy interval. For a continuous function $f: \mathbb{R}^{n} \rightarrow \mathbb{R}$, the $\alpha-$ cuts $\left[B_{\alpha}^{-}, B_{\alpha}^{+}\right]$of the fuzzy extension $\tilde{B}$ are obtained by solving the following box-constrained global optimization problems $(\alpha \in[0,1])$ :

$$
\begin{gathered}
B_{\alpha}^{-}=\min \left\{f\left(x_{1}, x_{2}, \ldots, x_{n}\right) \mid x_{k} \in\left[\tilde{A}_{k}\right]_{\alpha}, k=1,2, \ldots, n\right\} \\
B_{\alpha}^{+}=\max \left\{f\left(x_{1}, x_{2}, \ldots, x_{n}\right) \mid x_{k} \in\left[\tilde{A}_{k}\right]_{\alpha}, k=1,2, \ldots, n\right\} .
\end{gathered}
$$

For general functions, we need to solve numerically the global minimization and maximization problems above; it is clear that, except for simple cases, we have 
only the possibility of fixing a finite set of values $\alpha \in\left\{\alpha_{0}, \ldots, \alpha_{N}\right\}$ and obtain the corresponding $B_{\alpha}^{-}$and $B_{\alpha}^{+}$point-wise. To reduce these difficulties, various specific methods have been proposed (see, e.g., [16]), based on the fact that (1): all the minimization and the maximization problems have the same objective function, and (2): for different values of $\alpha$ the box-constraints are "nested", i.e., $\left[\tilde{A}_{k}\right]_{\alpha}$ contains $\left[\tilde{A}_{k}\right]_{\beta}$ for $\beta>\alpha$. To perform our computations, we have used the two procedures SPDE and MPDE, implemented using MATLAB, based on two special adaptations of the well-known Differential Evolution algorithm ( $D E$ for short) to the multiple-nested optimization problems (34) and 35); a detailed description with extended computational results can be found in Stefanini 26] and Stefanini et al. 32 .

The idea of $D E$ to find $\min$ or $\max$ of $\left\{f\left(x_{1}, \ldots, x_{n}\right) \mid\left(x_{1}, \ldots, x_{n}\right) \in \mathbb{A} \subset \mathbb{R}^{n}\right\}$ is relatively simple: start with an initial "population" $x^{(1)}=\left(x_{1}, \ldots, x_{n}\right)^{(1)}, \ldots$, $x^{(p)}=\left(x_{1}, \ldots, x_{n}\right)^{(p)} \in \mathbb{A}$ of $p$ feasible points and evolve the population of the current generation to obtain a new generation of points having better performances with respect to the objective function. To obtain a new generation, the actual points (individuals) are recombined randomly and new best points are selected to continue in the next generation. Denote by $x^{(k, g)}$ the $k$-th vector of the population at iteration (generation) $g$ and by $x_{j}^{(k, g)}$ its $j$-th component $(j=1, \ldots, n)$. At each iteration, the method generates a set of candidate points $y^{(k, g)}$ to substitute the elements $x^{(k, g)}$ of the current population, if $y^{(k, g)}$ is better. To generate $y^{(k, g)}$ two operations are applied: recombination and crossover. A typical recombination operates on a single component $j \in\{1, \ldots, n\}$ and generates a new perturbed vector of the form $v_{j}^{(k, g)}=x_{j}^{(r, g)}+\gamma\left[x_{j}^{(s, g)}-x_{j}^{(t, g)}\right]$, where $r, s, t \in\{1,2, \ldots, p\}$ are chosen randomly and $\gamma \in] 0,2]$ is a constant (eventually chosen randomly for the current iteration) that controls the amplification of the variation. The potential diversity of the population is controlled by a crossover operator, that constructs the candidate $y^{(k, g)}$ by crossing randomly 
the components of the perturbed vector $v_{j}^{(k, g)}$ and the old vector $x_{j}^{(k, g)}$ :

$$
y_{j}^{(k, g)}= \begin{cases}v_{j}^{(k, g)}, & \text { with probability } p \\ x_{j}^{(k, g)}, & \text { with probability } 1-p\end{cases}
$$

The candidate $y^{(k, g)}$ is then compared to the existing $x^{(k, g)}$ by evaluating the objective function at $y^{(k, g)}$ : if $f\left(y^{(k, g)}\right)$ is better than $f\left(x^{(k, g)}\right)$ then $y^{(k, g)}$ substitutes $x^{(k, g)}$ in the new generation $g+1$, otherwise $x^{(k, g)}$ is retained.

To take into account the particular nature of our problem, the basic $D E$ procedure is modified by two different strategies.

SPDE (Single Population DE procedure): start with the $(\alpha=1)-c u t$ back to the $(\alpha=0)-c u t$ so that the optimal solutions at a given level can be inserted into the "starting" populations of lower levels; use two distinct populations and perform the recombinations such that, during generations, one of the populations specializes to find the minimum and the other to find the maximum.

MPDE (Multi Populations DE procedure): use $2(N+1)$ populations to solve simultaneously all the box-constrained problems; $N+1$ populations specialize for the min and the others for the max and the current best solution for level $\alpha_{i}$ is valid also for levels $\alpha_{0}, \ldots, \alpha_{i-1}$.

A sufficiently precise calculation requires a number $N$ of $\alpha$-cuts in the range from 5 to 20, depending on the application and required precision (see Stefanini 26] for detailed computational results).

\subsection{Proposed Fuzzy Soil Model and Methodology}

The fuzzy soil model to use in the stochastic site response analysis is hereinafter determined. Moreover, the section proposes the methodology to construct the membership functions of the soil parameters in order to treat the uncertainty according to the fuzzy interpretation. Finally, the definition of gradual elastic response spectrum is established.

\subsubsection{Fuzzification of the stochastic site response analysis}

The fuzzy logic approach to the analysis entails a transformation process of the crisp (or deterministic) system parameters into fuzzy sets with grades 
of membership, referred to as fuzzification; different levels of fuzziness can be defined according to which parameters are considered as fuzzy sets and which are not, hence, being described by crisp or deterministic values. Each soil parameter that can be interpreted as a fuzzy value is indicated by a component, $\tilde{A}_{k}$, of the $n$-dimensional input vector $\tilde{\mathbf{A}}$. Therefore, the fuzzification process of Eq. (7), for a general layer, leads to the following hybrid fuzzy-stochastic equation:

$$
\tilde{S}_{U U}(\omega, \tilde{\mathbf{A}})=|\tilde{H}(\omega, \tilde{\mathbf{A}})|^{2} S_{U_{g} U_{g}}(\omega)
$$

in which $S_{U_{g} U_{g}}(\omega)$ is the input power spectral density, as a function of the circular frequency $\omega$, used in the stochastic formulation of Eq. $(7) ; \tilde{H}(\omega, \tilde{\mathbf{A}})$ is the fuzzy-valued transfer function where the system fuzzy parameters collected in $\tilde{\mathbf{A}}$ are made explicit. Therefore, the outcome of Eq. (37) is the hybrid fuzzystochastic power spectral density $\tilde{S}_{U U}$ defined as follows:

$$
\tilde{S}_{U U}: \omega \in \mathbb{R}^{+}, \tilde{\mathbf{A}} \in \mathcal{F}^{n} \longrightarrow \tilde{S}_{U U}(\omega, \tilde{\mathbf{A}}) \in \mathcal{F}^{1}
$$

It is important to consider that, as a function of $\omega \in \mathbb{R}^{+}$and for fixed fuzzy parameters $\tilde{\mathbf{A}}$, the function

$$
\omega \longrightarrow \tilde{S}_{U U}(\omega, \tilde{\mathbf{A}}) \in \mathcal{F}^{1}
$$

is fuzzy-valued and it is obtained by the application of the extension principle as in Eq. 38. On the other hand, our fuzzy output $\tilde{a}_{P G A}$, as described in Eq. 40. below, is itself obtained by the extension principle, as detailed in subsection 3.2.3: it is the fuzzy interval representing the median value $\tilde{a}_{P G A}$ of the largest peak of the acceleration at the top surface determined as

$$
\tilde{a}_{P G A}(\tilde{\mathbf{A}})=\tilde{\eta}_{\ddot{U}}(\tilde{\mathbf{A}}) \sqrt{\tilde{\lambda}_{0, \ddot{U}}(\tilde{\mathbf{A}})}
$$

in which $\tilde{\lambda}_{0, \ddot{U}}(\tilde{\mathbf{A}})$ is the fuzzy zeroth-order response spectral moment derived from Eq. 11) and $\tilde{\eta}_{\ddot{U}}(\tilde{\mathbf{A}})$ is the fuzzy peak factor at the ground level given by Eq. 13 after considering the soil-site properties of site response problem as fuzzy sets. In Eq. 40 , the zeroth-order response spectral moment, $\tilde{\lambda}_{0, \ddot{U}}$ as 
well as the $r-t h$ response spectral moments used to derive the peak factor of Eq. 13 , are defined in fuzzy terms as

$$
\tilde{\lambda}_{r, \ddot{U}}(\tilde{\mathbf{A}})=\int_{0}^{\infty} \omega^{4+r} \tilde{S}_{U U}(\omega, \tilde{\mathbf{A}}) \mathrm{d} \omega
$$

for $r=0,1,2$ and its membership function is determined as follows:

$$
\mu_{\tilde{\lambda}_{r}}(x)=\sup \left\{\alpha \mid x \in\left[\lambda_{r, \alpha}^{-}, \lambda_{r, \alpha}^{+}\right]\right\}
$$

It is worth mentioning that the integral of the set-valued function in Eq. 411 is intended in Aumann's sense [33] where, for $\alpha \in] 0,1]$, the $\alpha-$ cut of $\tilde{\lambda_{r}}$ is defined by

$$
\left[\tilde{\lambda}_{r}\right]_{\alpha}=\left[\int_{0}^{\infty} \omega^{4+r} \tilde{S}_{U U, \alpha}^{-} \mathrm{d} \omega, \int_{0}^{\infty} \omega^{4+r} \tilde{S}_{U U, \alpha}^{+} \mathrm{d} \omega\right] .
$$

Therefore, the formulation proposed in this paper of the site response analysis combines independently the uncertainty of the seismological event interpreted as a stochastic process with the uncertainty of the soil parameters intended as fuzzy intervals because of the lack of knowledge supplied by actual experience. Furthermore, the analysis provides information about the propagation of the epistemic and aleatory uncertainty on the ground surface motion.

\subsubsection{Construction of the soil parameter membership function}

In the present study, the method of constructing membership functions of the soil parameters collected in the vector $\tilde{\mathbf{A}}$ is based on knowledge acquisition procedure where the objective available information but imprecise due to limited number of laboratory and/or in-situ tests usually conducted to characterize the soil, is supplied by subjective information estimates on single expert or multiple experts opinions on the basis of engineering judgement (see, e.g., Pham and Valliappan [34]).

The proposed input reference membership function (see Figure 2 for the $k-$ th soil parameter, $\tilde{A}_{k}$, has an initial symmetric trapezoidal shape characterized by three features: i) the core mid-point $A_{k}$ of the parameter representing the "best-estimate" or crisp value, ii) the confidence, in non-probabilistic sense, or spread factor $\sigma_{\text {core }}$ of the core interval with respect to the core mid-point $A_{k}$, and 
iii) the left and right spread factors, $\sigma_{\text {supp }}^{L}$ and $\sigma_{\text {supp }}^{R}$, respectively. Accordingly, core and support intervals, representing the intervals for which the degree of belief is maximum and minimum, respectively, are defined as follows:

$$
\begin{gathered}
\operatorname{core}(\tilde{A})=\left[A_{k}\left(1+\sigma_{\text {core }}\right), A_{k}\left(1+\sigma_{\text {core }}\right)\right] \\
\operatorname{supp}(\tilde{A})=\left[A_{k}\left(1-\sigma_{\text {supp }}^{L}\right), A_{k}\left(1-\sigma_{\text {supp }}^{R}\right)\right]
\end{gathered}
$$

Furthermore, by using the notion of Eq. $31,, a=A_{k}\left(1-\sigma_{\text {supp }}^{L}\right), b=A_{k}(1-$ $\left.\sigma_{\text {core }}\right), c=A_{k}\left(1+\sigma_{\text {core }}\right)$, and $d=A_{k}\left(1-\sigma_{\text {supp }}^{R}\right)$. If a symmetric fuzzy input interval is assumed, $\sigma_{\text {supp }}^{L}=\sigma_{\text {supp }}^{R}$; nevertheless, the fuzzy output may result with a asymmetric membership function characterized by $\sigma_{\text {supp }}^{L} \neq \sigma_{\text {supp }}^{R}$.

Therefore, in order to fully define the membership function, $A_{k}, \sigma_{s u p p}$, and, $\sigma_{\text {core }}$ should be determined. In this paper, the support, characterized by $\sigma_{\text {supp }}^{L}$ and $\sigma_{\text {supp }}^{R}$, is estimated by experts opinions from engineering practical intervals given in literature or determined from previous experiences; since the support bounds those values that belongs to the set from those that does not belong to it, thus, it can be straightforwardly estimated from existing data on similar soils. The fuzzy core, characterized by $A_{k}$ and $\sigma_{c o r e}$, is considered an interval purely depending on the aleatory nature of the data which statistical propositions such as point best estimate and confidence interval; they are inferred by statistical analysis of the laboratory and in-situ geotechnical tests carried out for the investigated site-specific soil. It is worth emphasising that the linear trapezoidal shape represents the propagation of the epistemic uncertainty, decreasing with the increase of the $\alpha$-cut from $\alpha=0$ up to $\alpha=1$. Nevertheless, the hybrid fuzzy - stochastic 1D site response analysis proposed in this paper is not restricted to the above linear membership function but other techniques for generating nonlinear membership functions can be used (see, e.g., Pham and Valliappan, 34], Stefanini et al. [35]). 


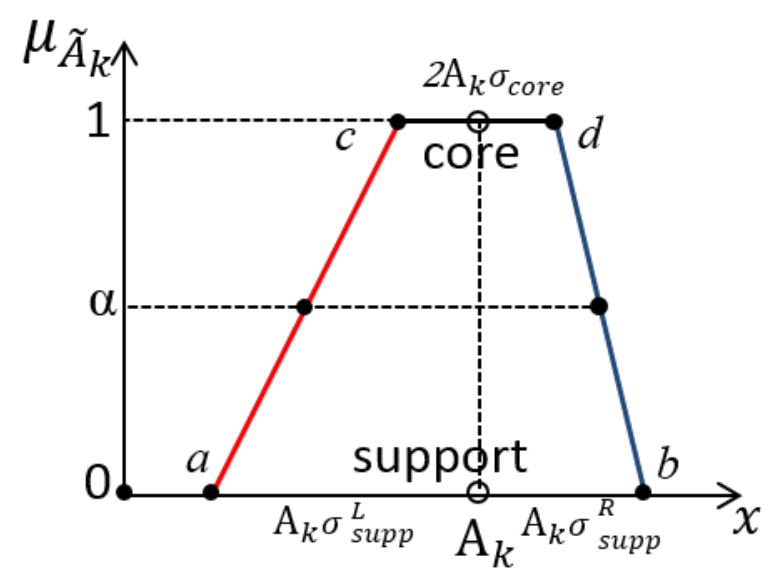

Figure 2: Generic trapezoidal membership function with linear LR sides

\subsubsection{Computational Approach}

Computational results are obtaining by applying the Zadeh's extension principle [14]. The hybrid fuzzy-stochastic outcome, defined as function $\tilde{a}_{P G A}(\tilde{\mathbf{A}})=$ $\tilde{f}\left(\tilde{A}_{1}, \tilde{A}_{2}, \ldots, \tilde{A}_{n}\right)$ of Eq. 40 , represents the fuzzy extension of the crisp function of Eq. (11), for $n$ fuzzy parameters, defined by the following membership function:

$$
\mu_{\tilde{a}_{P G A}}(x)=\sup \left\{\alpha \mid x \in\left[a_{P G A, \alpha}^{-}, a_{P G A, \alpha}^{+}\right]\right\} .
$$

Therefore, in order to generate the fuzzy output, the minimum $\tilde{a}_{P G A, \alpha}^{-}$and the maximum $\tilde{a}_{P G A, \alpha}^{+}$should be obtained for each $\alpha \in[0,1]$. In this paper, the SPDE algorithm is applied to the box-constrained global optimization problem:

$$
\begin{aligned}
& a_{P G A, \alpha}^{-}=\min \left\{f\left(x_{1}, \ldots, x_{n}\right) \mid x_{k} \in\left[\tilde{A}_{k}\right]_{\alpha}, k=1, \ldots, n\right\} \\
& a_{P G A, \alpha}^{+}=\max \left\{f\left(x_{1}, \ldots, x_{n}\right) \mid x_{k} \in\left[\tilde{A}_{k}\right]_{\alpha}, k=1, . ., n\right\}
\end{aligned}
$$

where $n$ is the total number of fuzzy parameters $\tilde{A}_{k}$ for $k=1, \ldots, n$ and (here $x_{j}$ denotes for simplicity the $j$-th patameter)

$$
f\left(x_{1}, \ldots, x_{n}\right)=\eta_{U}\left(x_{1}, \ldots, x_{n}\right) \sqrt{\lambda_{0, U}\left(x_{1}, \ldots, x_{n}\right)} .
$$


The optimization problem is solved numerically as described in section 3.1 by using a population $p$ of 200 members for each of the $N=11 \alpha$-cuts calculated in the analysis and a tolerance set to $1.0 E-5$. The $N-\alpha$-cuts are selected uniformly between 0 and $1, \alpha_{i}=\frac{i-1}{N-1}, i=1, \ldots, N$.

\subsubsection{Gradual Elastic Response Spectrum}

From the application of the extension principle to Eq. 40, for each value of the gradual scale $\alpha \in[0,1]$, we obtain the $\alpha$-cut $\left[a_{P G A, \alpha}^{-}, a_{P G A, \alpha}^{+}\right]$of the fuzzy-valued output $\tilde{a}_{P G A}(\tilde{\mathbf{A}})$; from the solutions of the two min and $\max$ optimization problems 47 and 48 let us denote by $A_{k, \alpha}^{-}$(for the min problems) and $A_{k, \alpha}^{+}$(for the max problems) the values of the optimal parameters $A_{k}$, $k=1, \ldots, n$. Corresponding to the vectors $\mathbf{A}_{\alpha}^{-}=\left(A_{1, \alpha}^{-}, \ldots, A_{n, \alpha}^{-}\right)$and $\mathbf{A}_{\alpha}^{+}=$ $\left(A_{1, \alpha}^{+}, \ldots, A_{n, \alpha}^{+}\right)$of parameters, the power spectral densities $S_{U U}\left(\omega, \mathbf{A}_{\alpha}^{-}\right)$and $S_{U U}\left(\omega, \mathbf{A}_{\alpha}^{+}\right)$are typical gradual functions (Dubois and Prade [36]), for each value of $\alpha \in[0,1]$ :

$$
\begin{gathered}
\omega \longrightarrow \hat{S}_{U U}\left(\omega, \mathbf{A}_{\alpha}^{-}\right) \in \mathbb{R} \\
\omega \longrightarrow \hat{S}_{U U}\left(\omega, \mathbf{A}_{\alpha}^{+}\right) \in \mathbb{R}
\end{gathered}
$$

and in general they are not fuzzy-valued intervals because they do not define a membership function; for each fixed $\alpha \in[0,1]$, the two functions above are standard power spectral density functions which define the lower and upper values of the fuzzy peak ground acceleration $\tilde{a}_{P G A}$.

Similarly, the optimal parameters, $\mathbf{A}_{\alpha}^{-}$and $\mathbf{A}_{\alpha}^{+}$can be applied to compute the gradual elastic response spectra, $\alpha \in[0,1]$ :

$$
\begin{aligned}
& T \longrightarrow \hat{S}_{a}\left(T, \mathbf{A}_{\alpha}^{-}\right) \in \mathbb{R} \\
& T \longrightarrow \hat{S}_{a}\left(T, \mathbf{A}_{\alpha}^{+}\right) \in \mathbb{R}
\end{aligned}
$$

providing the peak intervals of the response of the single oscillator (SDOF) system (see Eq. 23) used for the structural design. 
More precisely, the proposed gradual elastic response spectrum is obtained, for $\hat{\mathbf{A}}=\mathbf{A}_{\alpha}^{-}$or $\hat{\mathbf{A}}=\mathbf{A}_{\alpha}^{+}$, as

$$
\hat{S}_{a}(T, \hat{\mathbf{A}})=\eta_{\ddot{U}}(\hat{\mathbf{A}}) \sqrt{\hat{\lambda}_{0, T}(\hat{\mathbf{A}})}
$$

where:

$$
\hat{\lambda}_{0, T}(\hat{\mathbf{A}})=\int_{0}^{\infty} \omega^{4}\left|H_{S D O F}(\omega, T)\right|^{2} \hat{S}_{U U}(\omega, \hat{\mathbf{A}}) \mathrm{d} \omega .
$$

For a given value of the structural period $T$ and for a given vector of parameters $\hat{\mathbf{A}}$, the gradual elastic response spectrum $\hat{S}_{a}(T, \hat{\mathbf{A}})$ represents the pseudoacceleration of the SDOF system (see Eq. 24). According to Fortin et al. [37, the quantities in Eq. 52 and Eq. 53 are families of gradual numbers, for a fixed value of the structural period $T$, that correspond to the values $\mathbf{A}_{\alpha}^{-}$and $\mathbf{A}_{\alpha}^{+}$of the parameters which produce the extreme values $a_{P G A, \alpha}^{-}$and $a_{P G A, \alpha}^{+}$of the $\alpha$-cuts of our fuzzy output $\widetilde{a}_{P G A}(\widetilde{\mathbf{A}})$. In the following Section 4 graphical representations of the gradual power spectral density functions of Eq. 52 and Eq. 53 and of the gradual elastic response spectra of Eq. 50 and Eq. 51 as well as their interpretation will be discussed.

\section{Case Studies}

Hybrid fuzzy-stochastic 1D site response analyses are carried out by considering the approach proposed in the previous Section 3 Four different soil deposits classified into ground types A-B-C-D complying with the seismic code EN 1998-1 [15] are investigated. The soil profiles consist of saturated clays with different consistency, ranging from rigid to soft, each one characterized by mass density $\rho$ of $2100 \mathrm{~kg} \mathrm{~m}^{-3}$ and Poissons ratio $\nu$ of 0.45 and resting upon a uniform linear visco-elastic bedrock. The mechanical parameters of the soil deposits and the underlying bedrock, intended as crisp or "best-estimate" values, are reported in Table 1 where the crisp shear wave velocity of the soil, calculated

according to the relation $V_{s}=\sqrt{\frac{G_{0}}{\rho}}$, is used to classify the soil in accordance to [15.

The input seismic process applied at the outcrop bedrock is fully described by 


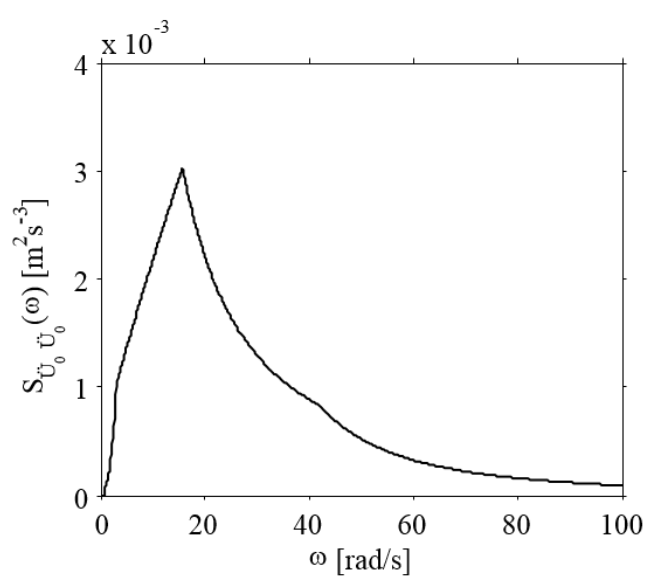

Figure 3: Power spectral density function defined at the outcrop bedrock

the stationary power spectral density in acceleration, $S_{\ddot{U}_{0} \ddot{U}_{0}}(\omega)=\omega^{4} S_{U_{0} U_{0}}(\omega)$, determined from the response-spectrum-compatible model of Cacciola et al. 38] depicted in Figure 3, consistent with the soil type A and peak ground acceleration $a_{0}=0.96 \mathrm{~m} \mathrm{~s}^{-2}$.

The initial homogeneous profile is discretized in $0.5 \mathrm{~m}$ thick-layers assuming constant equivalent linear properties, compatible with the current shear strain computed at each iteration, according to the modulus reduction curve and the damping ratio curve proposed by Seed and Sun [39] and Idriss [40, respectively, as depicted in Figure 4 in black colour.

Table 1: Crisp soil parameters

\begin{tabular}{cccccc}
\hline Ground type & $V_{s}[\mathrm{~m} / \mathrm{s}]$ & $G_{0}[\mathrm{MPa}]$ & $\rho\left[\mathrm{kg} / \mathrm{m}^{3}\right]$ & $\nu$ & $\xi_{f}$ \\
\hline A/bedrock & 1000 & $2.1 \times 10^{6}$ & 2100 & 0.45 & 0.1 \\
B & 400 & $3.36 \times 10^{5}$ & 2100 & 0.45 & 0.15 \\
$\mathrm{C}$ & 250 & $1.3 \times 10^{5}$ & 2100 & 0.45 & 0.28 \\
$\mathrm{D}$ & 150 & $4.7 \times 10^{4}$ & 2100 & 0.45 & 0.30 \\
\hline
\end{tabular}

The investigated uncertainties are the position of the bedrock that determines the soil deposit thickness $h$, the initial shear modulus $G_{0}$, the unit den- 
Table 2: Fuzzy sets parameters

\begin{tabular}{cccc}
\hline Fuzzy set & Core mid-point & $\sigma_{\text {core }}$ & $\sigma_{\text {supp }}^{L, R}$ \\
\hline$\tilde{h}$ & $40 \mathrm{~m}$ & 0.00 & 0.2 \\
$\tilde{G_{0}}$ & $(210,33.6,13.0,4.7) \times 10^{4} \mathrm{MPa}$ & 0.05 & 0.3 \\
$\tilde{\rho}$ & $2100 \mathrm{~kg} \mathrm{~m}^{-3}$ & 0.02 & 0.2 \\
$\tilde{\xi_{f}}$ & $0.1,0.15,0.28,0.3$ & 0.02 & 0.1 \\
\hline
\end{tabular}

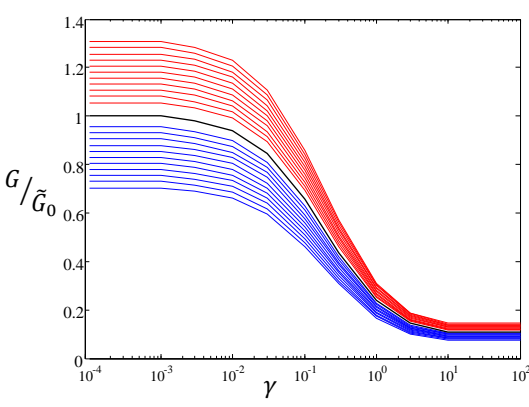

(a)

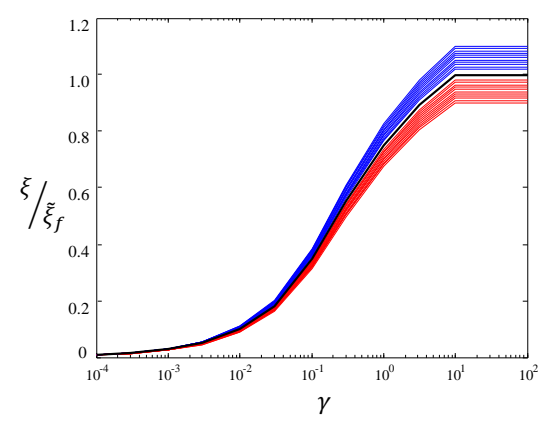

(b)

Figure 4: a) Shear modulus degradation curve and b) critical damping ratio curve proposed by Seed and Sun [39] and Idriss [40] in black colour and their fuzzy interpretation

sity, $\rho$, as well as the maximum critical damping ratio $\xi_{f}$ expressed as fuzzy sets, i.e. $\tilde{h}, \tilde{G}_{0}, \tilde{\rho}$ and $\tilde{\xi_{f}}$, respectively. Based on subjective information, symmetric trapezoidal-shaped membership functions are constructed for describing the uncertain parameters for the four soil deposits considered in the analysis; Table 2 reports the parameters that determine each membership function. The fuzzy set $\tilde{h}$ has $\sigma_{\text {core }}=0$, thus it is a symmetric triangular fuzzy number whose core is crisp because of a sharp interface boundary is assumed. Remarkably, the investigated soil deposits, classified by considering the crisp value of each soil properties, have different level of membership for each of the four types of soil defined in the seismic code [15]; therefore, they might be assigned to different rank as a function of the level of $\alpha$-cut that has been considered.

The simulation is carried out by developing a numerical algorithm in MAT- 
LAB environment used to solve the $\alpha$-cut problem at $11 \alpha$-cuts; five of them, namely $\alpha=0,0.25,0.5,0.75$ and 1 are highlighted in order to associate them to a linguistic ranking of knowledge (scarce, medium, sound, good, exhaustive). Differential evolution method is applied in order to obtain the fuzzy extension of the function as defined in Eq. (40). Noteworthy, the result of the partial differential equation is the power spectral density, hence a functional depending on frequency and fuzzy variables; therefore in order to deal with the optimization procedure of Eq. (47) and Eq. (48), a parameter of synthesis is defined. In particular, in this paper, results of problem solving are presented in terms of fuzzy set of the median value $\tilde{a}_{P G A}$ defined in Eq. 40 for each of the four soil deposits. Finally, the analysis aims to evaluate the response of the single oscillator (SDOF) constructed by extracting points from the previous elastic response spectrum at the fundamental period of $T=0.5 \mathrm{~s}$.

\subsection{Soil Type A}

The fuzzy input membership functions of the soil type A used in the analysis are depicted in Figure $5 a$ while Figure 5 b shows the result of the analysis in terms of membership function of the median peak ground surface acceleration,

$\tilde{a}_{P G A}$. It is worth mentioning that each black dot on the left and right side of the membership function is given by a crisp quadruple of values, $\left(h, G_{0}, \xi_{f}, \rho\right)$, belonging to the pertinent $\alpha$-cut interval of each fuzzy input, through the optimization problems (min and $\max$ ) of Eq. 40p; i.e., for each $\alpha \in[0,1]$, two quadruples of values $h \in[\tilde{h}]_{\alpha}, G_{0} \in\left[\tilde{G}_{0}\right]_{\alpha}, \rho \in\left[\tilde{\rho}_{0}\right]_{\alpha}$, and $\xi_{f} \in\left[\tilde{\xi}_{f}\right]_{\alpha}$, corresponding to the minimization and the maximization problems, are obtained. The result in Figure 5b shows that the core mid-point value is exactly corresponding to the peak input ground acceleration $a_{0}=0.96 \mathrm{~m} \mathrm{~s}^{-2}$, since the soil deposit is characterized by the same properties as the outcropping bedrock. The fuzzy output has a nonlinear LR-shaped membership function, fairly asymmetric with respect to the core towards the right branch; this is indicated by the dotted black curve collecting the mid-points of each $\alpha$-cut interval. Therefore, a higher uncertainty on the input parameters, namely a small value of $\alpha$-cut, 
leads to an overestimate of the expected, possible, peak ground acceleration on the ground surface with respect to the mid-core value. In Table 3 are reported the parameters of the membership functions resulting from the analysis in terms of core mid-point $a_{P G A}$ as well as core and support confidences $\sigma_{\text {core }}$ and $\sigma_{\text {supp }}^{L, R}$, respectively. In soil type $\mathrm{A}$, the measured global confidences of both the core and support of the result are lower than maximum values of confidences assumed for describing the fuzzy uncertainties of the input soil parameters. Therefore, the reduced degree of the uncertainty indicates a small sensitivity of the seismic response for this type of ground.
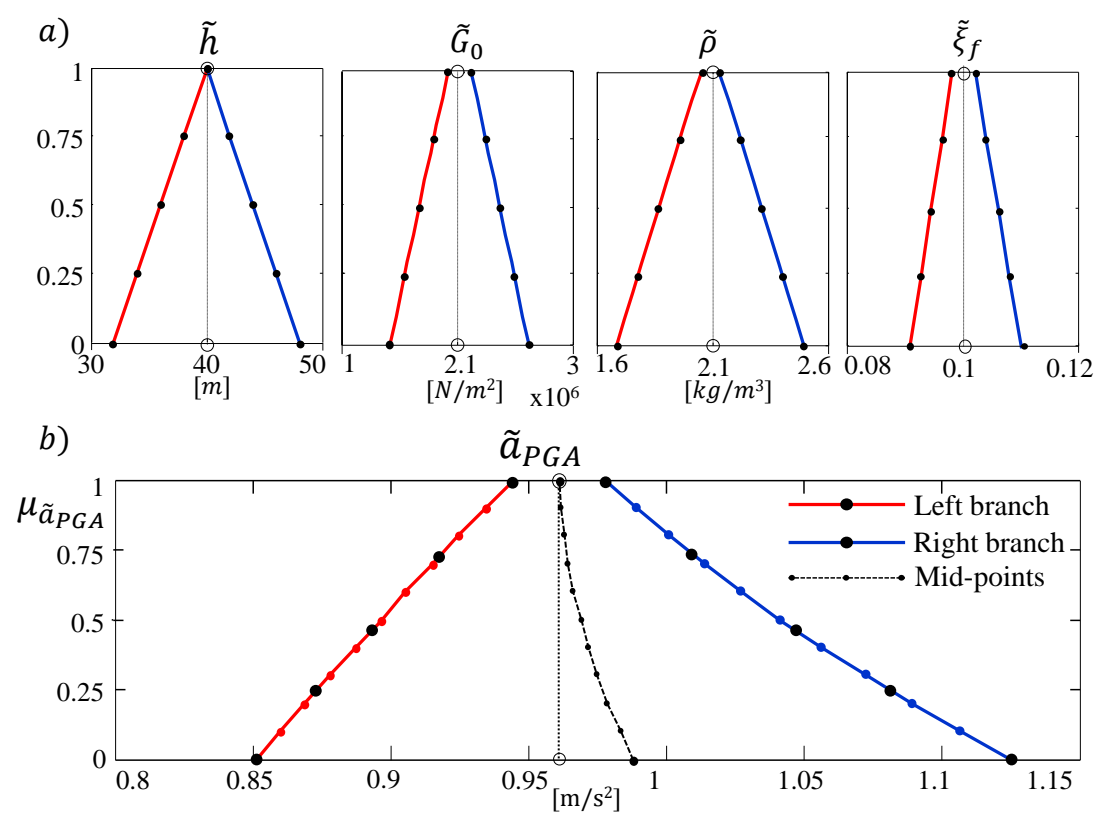

Figure 5: Membership function of the surface peak ground acceleration for ground soil A; (a) input fuzzy parameters and (b) fuzzy output.

Moreover, Figure 6(a) shows the power spectral density functions $S_{\ddot{U} \ddot{U}}$ associated to the fuzzy output through Eq. (37) where the relation, $S_{\ddot{U} \ddot{U}}(\omega)=$ $\omega^{4} S_{U U}(\omega)$ is used. Level curves are related to the edges of the interval for each $\alpha$-cut of the output; red and blue colours are used to distinguish the left- and right- side of the fuzzy output $\tilde{a}_{P G A}$ whereas a grey area collects all the points 
lying in the core interval. Finally, the power spectral density function related to the core mid-points of the soil properties, or crisp values $A_{k}$ previously defined in Table 1, is indicated by black colour; due to the stiff soil properties of the soil type A, this corresponds to the input power spectral density of Figure 3 It is worth noting that the predominant frequency does not greatly change by varying the $\alpha$-cut; the initial shape obtained by the crisp values, is scaled up in case of the upper branch or scaled down, in case of the lower branch, with the decrease of the $\alpha$-cut. By using the power spectral density functions previously determined, gradual elastic response spectra, depicted in Figure $7(\mathrm{a})$ are thus derived by applying Eq. (54). In order to study the effect of the uncertainty on the response in acceleration of a single oscillator, a section-cut of the elastic response spectra at the constant period $T=0.5 \mathrm{~s}$ is taken; Figure 7(b) shows the gradual maximum response acceleration as a function of the unit interval $[0,1]$ as described in Section 3.2.4. The two branches are almost symmetric with respect to the constant value determined from the crisp values and the propagation of uncertainty is slightly nonlinear; the main parameters describing the gradual intervals are reported in Table 4

\subsection{Soil Type B}

Fuzzy input membership functions for the investigated soil type B are depicted in Figure 8 a obtained by using the values reported in Table 2. The result of the analysis in terms of membership function of the median peak ground surface acceleration is illustrated in Figure 8b. The fuzzy output has a nonlinear LR-shaped membership function, asymmetric with respect to the core towards the right branch as indicated by the dotted black curve resulting in overrating

the expected peak ground acceleration on the ground surface with respect to the mid-core value. Moreover, as the previous case, the uncertainty evaluated through the spread factors reported in Table 3 , is reduced with respect to the uncertainty assumed for the input parameters of Table 2.

Figure 6(b) shows the power spectral density functions $S_{\ddot{U} \ddot{U}}$ associated to the fuzzy output through Eq. (37). The input PSD is altered by the soil working 


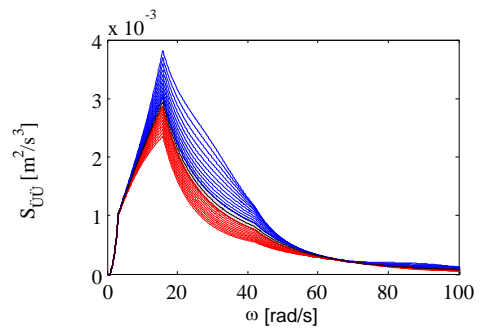

(a) \

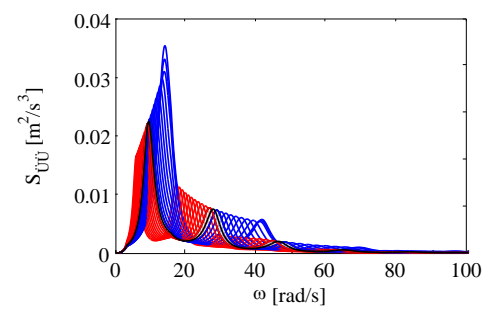

(c) 凹

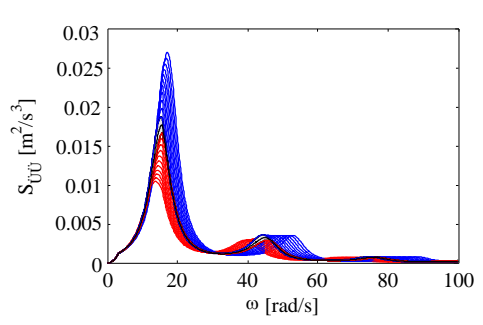

(b) \

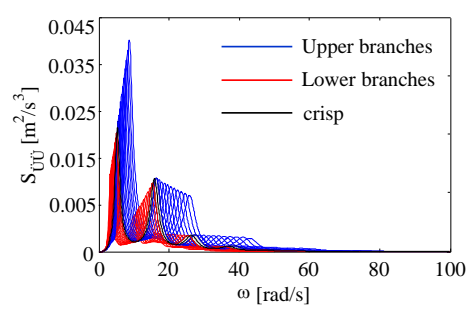

(d)

Figure 6: Fuzzy power spectral density for ground type (a) A, (b) B, (c) C, and (d) D.

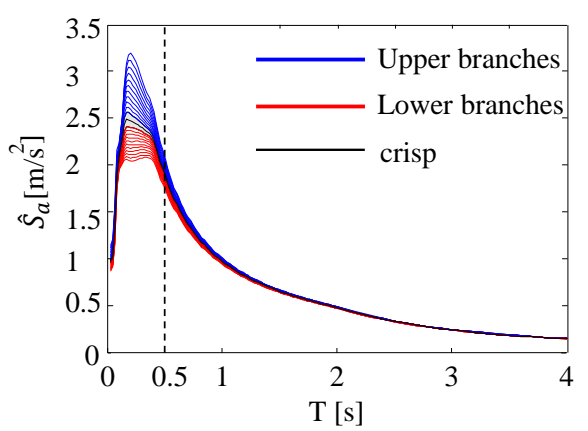

(a) 【

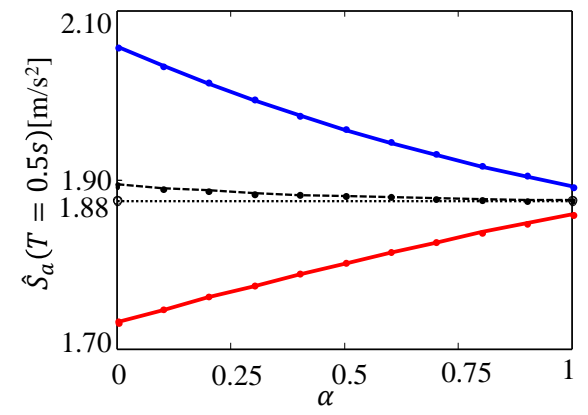

(b) ॥

Figure 7: Gradual Elastic Response Spectum (a) for soil type A and (b) pseudo-acceleration response in gradual interval representation. 

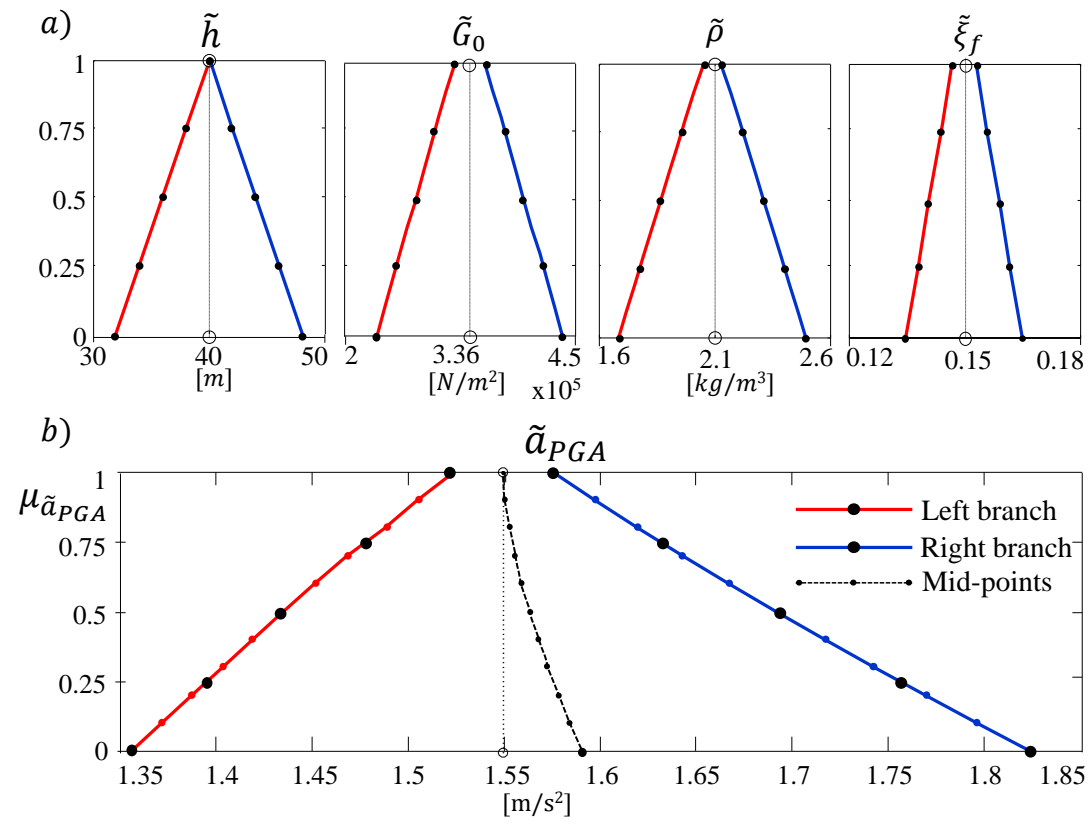

Figure 8: Membership function of the surface peak ground acceleration for ground soil B; (a) input fuzzy parameters and (b) fuzzy output

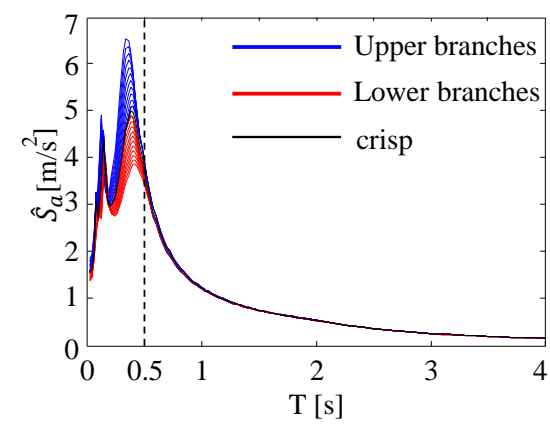

(a) 【

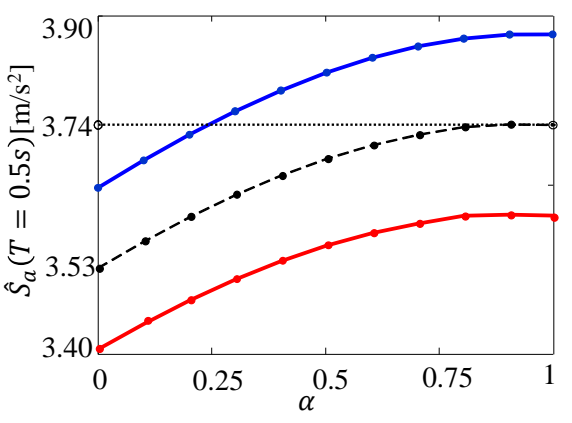

(b) 凹

Figure 9: Gradual Elastic Response Spectum (a) for soil type B and (b) pseudo-acceleration response in gradual interval representation. 
as a filter and the peaks of the response occur at the predominant frequencies of the soil deposit; especially, the uncertainty greatly affects the peak related to the second natural frequency where a large plateau given by the locus of local maximum value for every $\alpha$-cut, is observed.

By using the power spectral density functions previously achieved, the gradual elastic response spectra are determined in Figure 9(a). It is worth mentioning the response of the investigated SDOF illustrated in Figure 9(b) that shows that the support does not include all the core interval but even the mid-core value lies outside the support; this behaviour is clearly incompatible with the fuzzy algebra and this points out the diversity of the gradual intervals with respect to the fuzzy sets. Furthermore, the propagation of uncertainty is highly nonlinear and there is a decrease of the acceleration with the increase of lack of knowledge about the input parameters. This can lead to an underestimation of the actual response and hence, to a non-safe design. It is worth mentioning that the uncertainty in the values of the parameters $h, G_{0}, \rho$ and $\xi_{f}$ does not affect directly the value of $S_{a}$ at the specific period $T=0.5 \mathrm{~s}$, but the entire elastic response spectrum as a function of $T$ (i.e., changing $\alpha$ will change the function at all values of $T$ ). For the chosen $T=0.5 \mathrm{~s}$, all the gradual spectral functions of Eq. (54), remarkably the upper branches, have less uncertainty than the same spectra evaluated at different values of $T<0.5 s$ (e.g., $T=0.3 s$ ) on the left of the vertical dashed line in Figure 9(a) As it can be seen in Table 4 the maximum uncertainty with respect the crisp value is around $9 \%$.

\subsection{Soil Type $C$}

The investigated soil type $\mathrm{C}$ is characterized by the fuzzy soil properties depicted in Figure 10a. The result of the analysis in terms of membership function of the median peak ground surface acceleration is illustrated in Figure $10 \mathrm{~b}$. The membership function of the fuzzy output is strongly nonlinear, asymmetric with respect to the core towards the right branch resulting in overrating the expected peak ground acceleration on the ground surface with respect to the mid-core value. It can be observed from the dotted black curve that the propagation of 

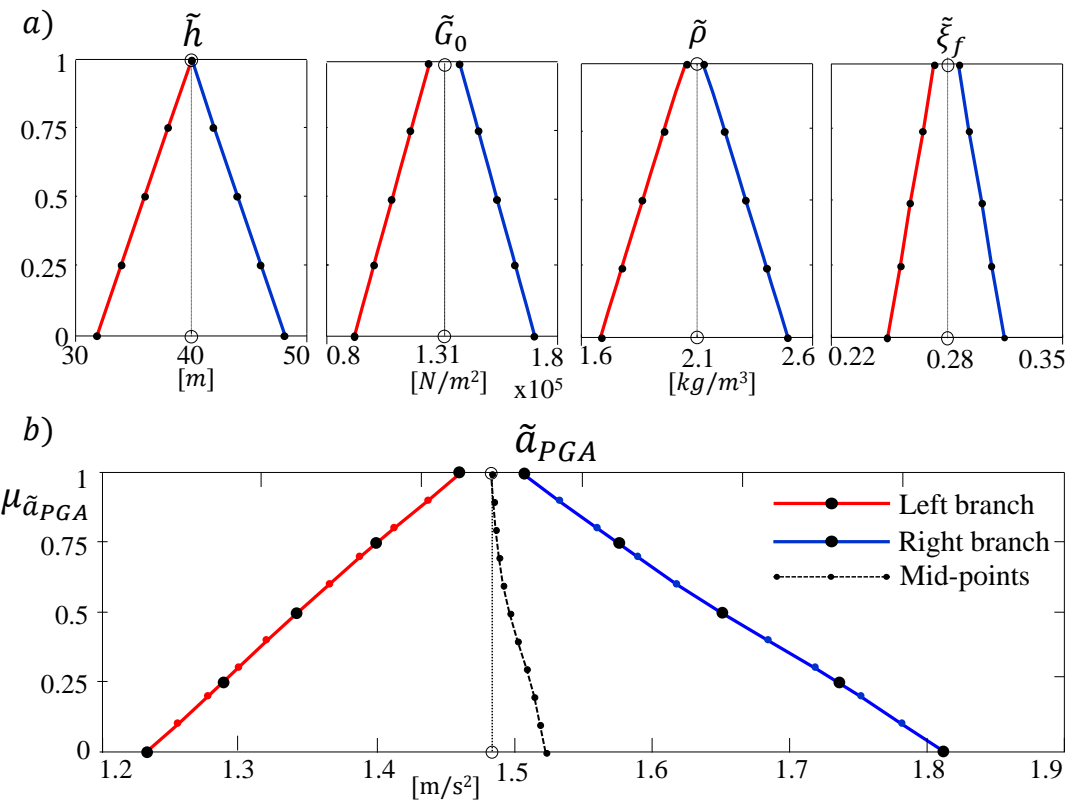

Figure 10: Membership function of the surface peak ground acceleration for ground soil C; (a) input fuzzy parameters and (b) fuzzy output. 
the uncertainty is associated with a change of the slope at around $\alpha$-cut $=0.5$. Moreover, as shown Table 3 the maximum value of the confidence of the support is as high as the mean confidence assumed for the input parameters.

Figure 6(c) shows the power spectral density functions $S_{\ddot{U} \ddot{U}}$ associated to the fuzzy output through Eq. (37). It is worth empathizing that the peaks of the response associated to the natural frequencies of the soil deposit cannot be observed since the initial peaks related to the crisp values are shifted in both directions by generating an envelope of the power spectral density without clear maxima; therefore, the uncertainty of the input parameters greatly affects the overall response.

As done for the previous cases, the elastic response spectra, shown in Figure 11(a) are obtained. By investigating the response of the single oscillator with fundamental period of $T=0.5 \mathrm{~s}$, the maximum pseudo-acceleration is computed as gradual interval in Figure 11(b), This shape is highly nonlinear and it can be observed an increase of the acceleration with the increase of the uncertainty of the input parameters. As reported in Table 4 , the uncertainty on the seismic response for the SDOF is relevant, reaching the maximum confidence of around $57 \%$ with respect the mid-core value.

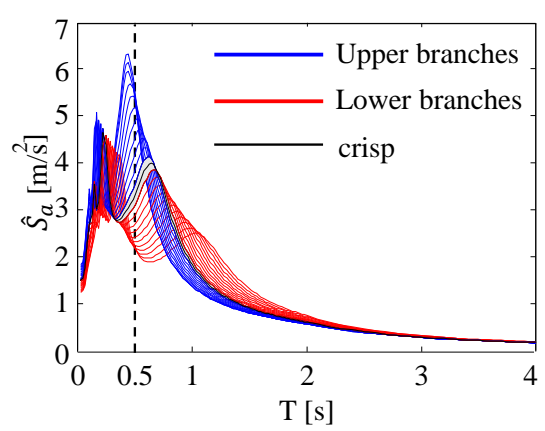

(a) \

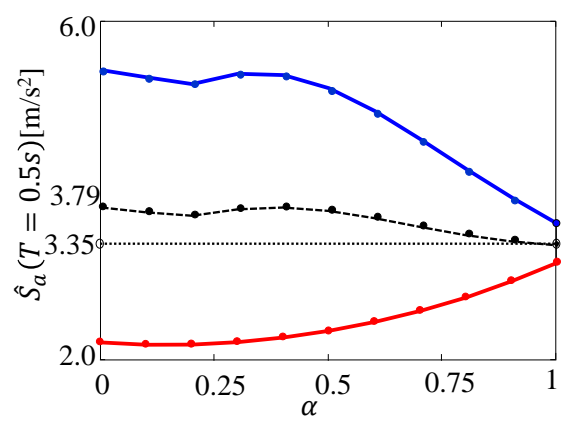

(b) 凹

Figure 11: Gradual Elastic Response Spectum (a) for soil type C and (b) pseudo-acceleration response in gradual interval representation. 

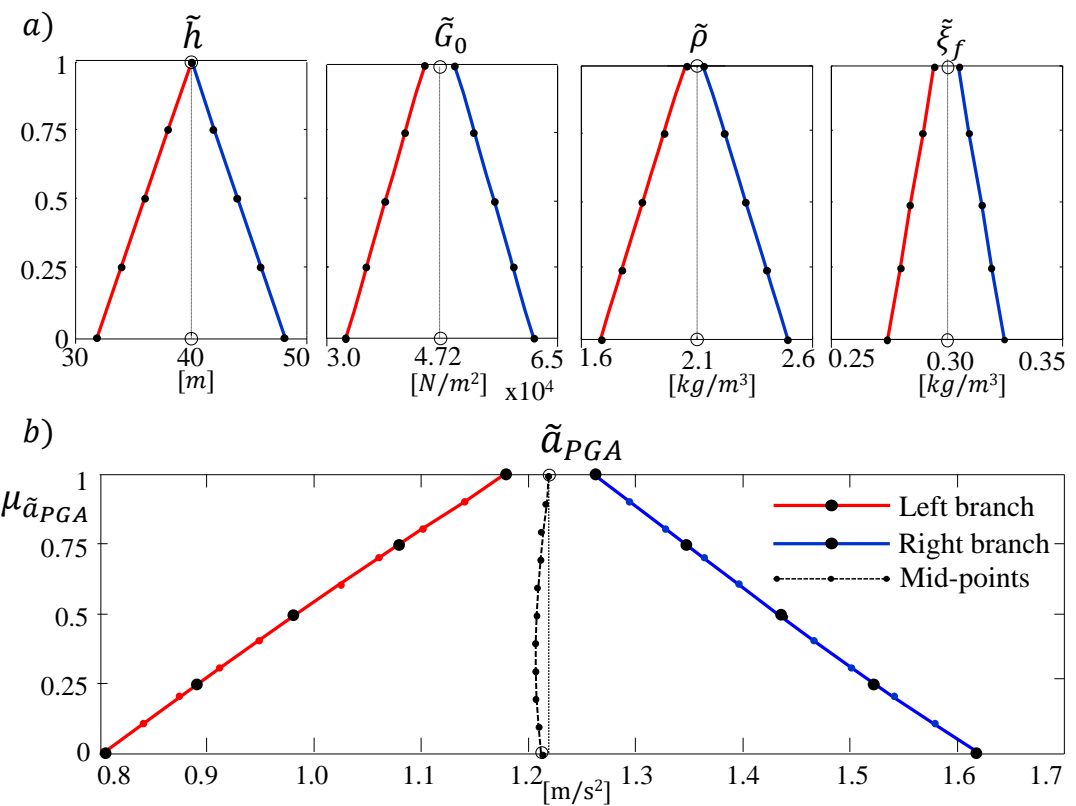

Figure 12: Membership function of the surface peak ground acceleration for ground soil D; (a) input fuzzy parameters and (b) fuzzy output. 


\subsection{Soil Type D}

The result of the fuzzy optimisation carried out by considering the fuzzy input membership functions reported in Figure $12 \mathrm{a}$ for the last investigated soil type $\mathrm{D}$ is depicted in Figure $12 \mathrm{~b}$; the membership function of the median peak ground surface acceleration is characterized by fairly symmetric trapezoidal shape but converse to the previous case, the values of the mid-points are smaller than the mid-core value inducing an underestimate of the expected, possible, peak ground acceleration on the ground surface with respect to the crisp, deterministic value.

Furthermore, in soil type D, the measured global confidences of the support of the result are higher than the average confidence values assumed for describing the fuzzy uncertainties of the input soil parameters, reaching a value around $30 \%$ as shown in Table 3. Therefore, an important sensitivity of the seismic response is expected for this type of ground.

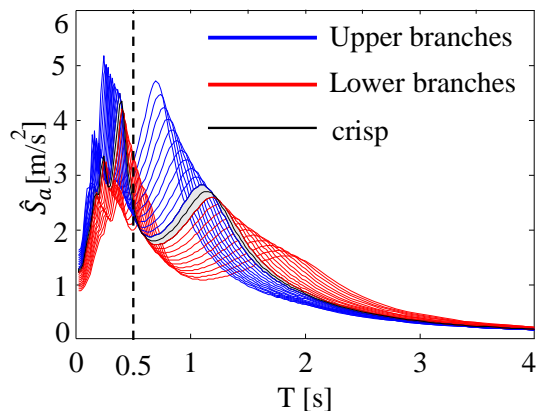

(a) 凹

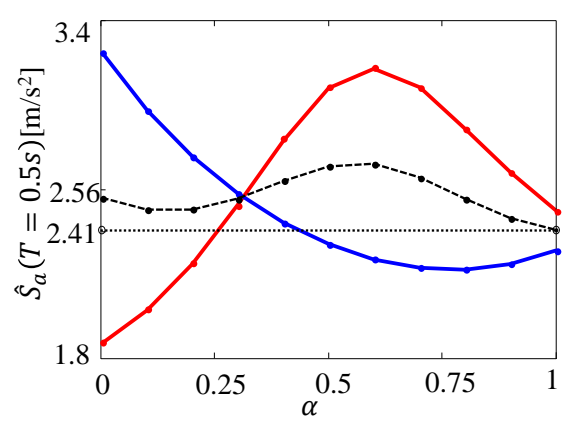

(b) ॥

Figure 13: Gradual Elastic Response Spectum (a) for soil type D and (b) pseudo-acceleration response in gradual interval representation.

Figure $6(\mathrm{~d})$ shows the power spectral density functions $S_{\ddot{U} \ddot{U}}$ associated to the fuzzy output through Eq. (37). As in the previous case, the uncertainty of the input parameters greatly affects the overall response, resulting in an envelope of the power spectral density function without exhibiting any punctual peaks.

Finally, in order to illustrate better the effects of the soil uncertainty on the 


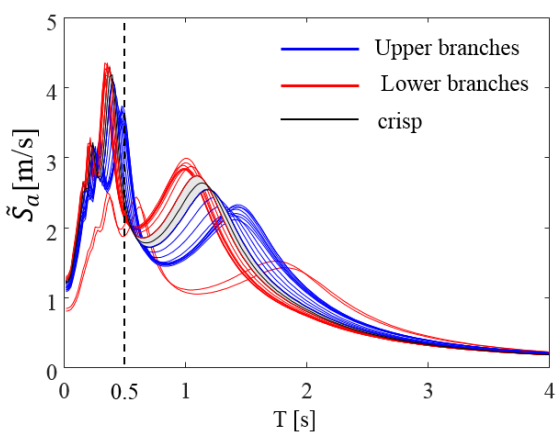

(a) 【

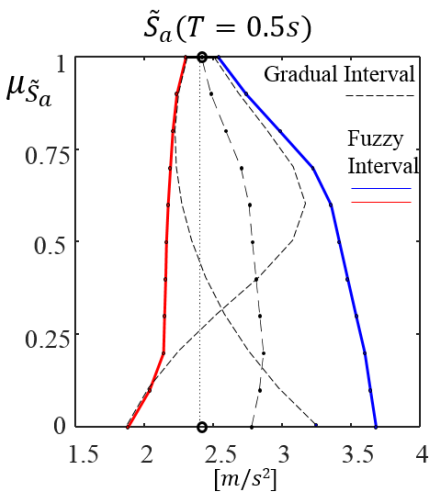

(b) \

Figure 14: Membership function of the Elastic Response Spectum (a) for soil type D and (b) fuzzy pseudo-acceleration response.

structural seismic response, the stochastic elastic response spectrum expressed as gradual function $S_{a}$ is hence determined. Regarding the structural period selected as $\mathrm{T}=0.5 \mathrm{~s}$, the gradual interval of the maximum pseudo-acceleration is extracted out from Figure 13(a) and depicted in Figure 13(b) Remarkably, the shape is highly nonlinear and non-monotonic; the functions obtained by the left and right branches of the fuzzy output, i.e. minimum and maximum $\tilde{a}_{P G A}$, respectively, are crossed each other with the increase of the membership value $\alpha$ in the input parameters; therefore, input soil parameters that provide the minimum peak acceleration at the ground level can produce the highest structural acceleration, and vice-versa. Moreover, the highest pseudo-accelerations does not occur only at the support but for different $\alpha$-cuts, e.g. with $\alpha=0.5$. Furthermore, from Figure 13(a) it can be seen that for this type of the soil, the gradual elastic response spectra remains strongly affected by uncertainty also for large values of the structural period $T$ (up to $T=2.5 \mathrm{~s}$ ) with a high variability of the structural acceleration and this strongly affects the engineering design. It is worth mentioning that the peak ground acceleration, used in this paper as parameter of synthesis of the box-constrained optimization approach proposed in Section 3.2 .3 is one of the main ground motion parameters con- 
sidered by the current seismic codes (see, e.g., EN 1998-1, [15]). Nevertheless, as it can be observed from the gradual interval in Figure 13(b) the maximum or minimum peak ground acceleration does not always lead to a corresponding maximum or minimum structural response. Therefore, in order to obtain the extreme structural responses, the structural pseudo-acceleration (e.g. at $\mathrm{T}=$ $0.5 \mathrm{~s}$ ) may be defined as parameter of synthesis in the optimization procedure of the hybrid stochastic-fuzzy analysis proposed in Eq. 477) and Eq. 480; in this case, the outcome of the structural response for the soil type D, is hence expressed in terms of fuzzy functions and fuzzy intervals and depicted in Figure 14(a) and Figure 14(b) respectively. In Figure 14(b), the achieved fuzzy interval is compared to the previous gradual interval obtained in Figure 13(b) for the same soil type showing that the gradual interval contains only a subset of the possible extreme structural accelerations that can be achieved by the combination of the soil parameters; therefore, the characterization of the ground motion only through the peak ground acceleration parameter can lead to underdesigned structures, while other ground motion parameters such as frequency content (see e.g. Greco et al. 41]) and duration (see e.g. Tombari et al. 42, Greco et al. [43 ) or a different parameter of synthesis based on structural characteristics have to be considered in order to perform a reliable structural analysis.

Table 3: Parameters of the output membership functions for the investigated soils.

\begin{tabular}{ccccc}
\hline Ground type & $a_{P G A}$ & $\sigma_{\text {core }}$ & $\sigma_{\text {supp }}^{L}$ & $\sigma_{\text {supp }}^{R}$ \\
\hline $\mathrm{A}$ & $0.960 \mathrm{~m} / \mathrm{s}^{2}$ & 0.017 & 0.115 & 0.171 \\
$\mathrm{~B}$ & $1.549 \mathrm{~m} / \mathrm{s}^{2}$ & 0.017 & 0.126 & 0.178 \\
$\mathrm{C}$ & $1.483 \mathrm{~m} / \mathrm{s}^{2}$ & 0.015 & 0.169 & 0.222 \\
$\mathrm{D}$ & $1.218 \mathrm{~m} / \mathrm{s}^{2}$ & 0.035 & 0.341 & 0.329 \\
\hline
\end{tabular}

\subsection{Sensitivity Analysis}

Finally a sensitivity study is carried out in order to evaluate the contribution of each fuzzy soil parameter on the response of the site response analysis in 
Table 4: Parameters of the output gradual intervals of the SDOF response for the investigated soils.

\begin{tabular}{ccccc}
\hline Ground type & $a_{P G A}$ & $\sigma_{\text {crisp }}$ & $\sigma_{\text {supp }}^{L}$ & $\sigma_{\text {supp }}^{R}$ \\
\hline $\mathrm{A}$ & $1.876 \mathrm{~m} / \mathrm{s}^{2}$ & 0.009 & 0.077 & 0.097 \\
$\mathrm{~B}$ & $3.738 \mathrm{~m} / \mathrm{s}^{2}$ & 0.036 & 0.089 & -0.025 \\
$\mathrm{C}$ & $3.352 \mathrm{~m} / \mathrm{s}^{2}$ & 0.068 & 0.350 & 0.614 \\
$\mathrm{D}$ & $2.409 \mathrm{~m} / \mathrm{s}^{2}$ & 0.039 & 0.221 & 0.349 \\
\hline
\end{tabular}

terms of fuzzy median acceleration at the top surface. The sensitivity analysis consists in the same type of analysis previously carried out where only one out of the four parameters at the time, $\left(\tilde{h}, \tilde{G}_{0}, \tilde{\rho}, \tilde{\xi_{f}}\right)$, is considered uncertain, in particular fuzzy, whereas the remaining others are kept as crisp values. The analysis is performed for the two types of soil that shown a higher impact of the soil uncertainty during the previous parametric analysis, namely soil type $\mathrm{C}$ and D. The same degree of uncertainty previously described in Table 2 is considered. Results are reported in terms of $\tilde{a}_{P G A}$ in Figure 15 and Figure 16 for soil type $\mathrm{C}$ and $\mathrm{D}$, respectively. It is worth noting that the uncertainty on the damping property, $\tilde{\xi_{f}}$ is less important than the other parameters $\left(\tilde{h}, \tilde{G}_{0}\right.$, and $\left.\tilde{\rho}\right)$ that affect almost equally the seismic response. These three parameters affect the natural frequencies of the soil deposit and thus, the frequency content of its transfer function. In particular, the uncertainty on $\tilde{G}_{0}$ has the highest impact on the response as seen from the larger support of the fuzzy output. Moreover, the sensitivity study has shown a non-symmetric and non-linear propagation of the uncertainty with most of the cases presenting a tendency in underestimating of the crisp result with the decrease of the $\alpha$-cut level except for the case related to the uncertainty on $\tilde{G}_{0}$.

\section{Concluding Remarks}

A fuzzy logic approach for dealing with soil uncertainties has been applied to the stochastic 1D site response analysis. The proposed hybrid probabilistic 


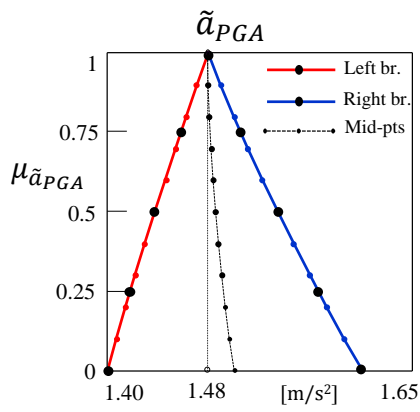

(a) «

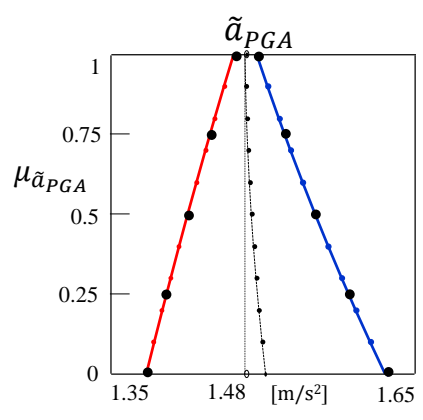

(c)

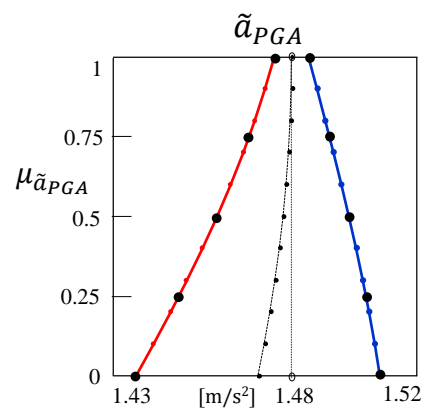

(b) 凹

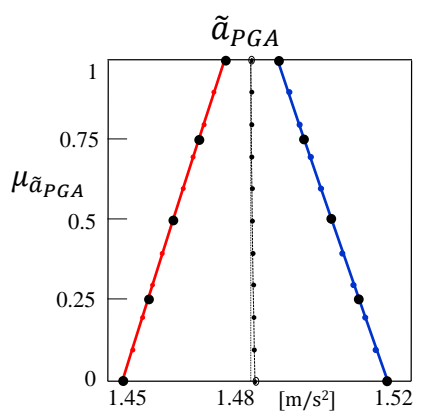

(d) 凹

Figure 15: Fuzzy Sensitivity analysis for soil type C by varying (a) $\tilde{h},(\mathrm{~b}) \tilde{G_{0}}$, (c) $\tilde{\rho}$, and (d) $\left.\tilde{\xi_{f}} \cdot\right)$ 


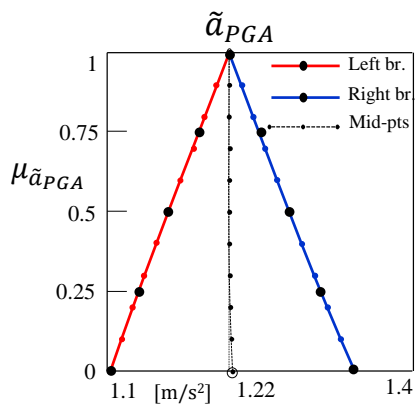

(a) «

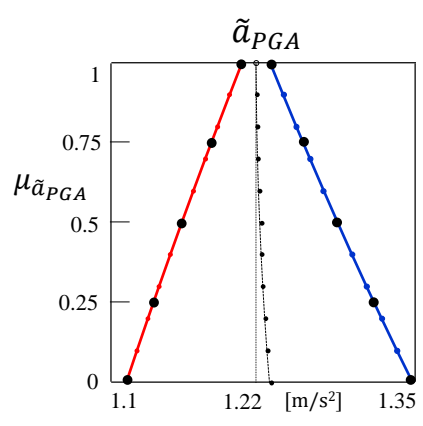

(c) 【

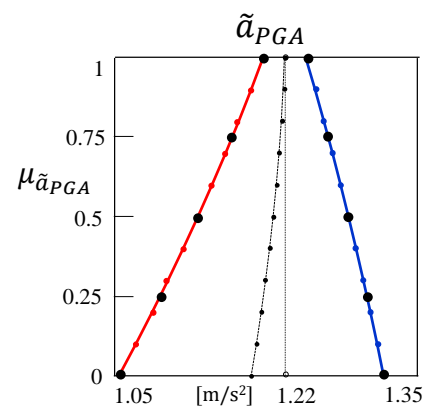

(b) 凹

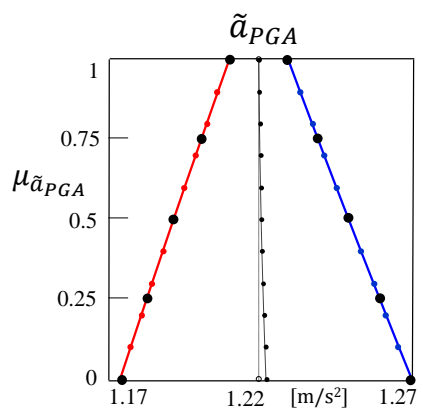

(d) 凹

Figure 16: Fuzzy Sensitivity analysis for soil type D by varying (a) $\tilde{h},(\mathrm{~b}) \tilde{G}_{0},(\mathrm{c}) \tilde{\rho}$, and (d) $\tilde{\xi_{f}}$. 
and fuzzy approach is used when soil uncertainties derived from a genuine lack of knowledge due to the few amount of data obtained from geotechnical surveys. Therefore, the soil parameters have been expressed in terms of fuzzy sets defined by symmetric trapezoidal shaped membership functions, assuming the independence between the inputs (see e.g. 44]). In this paper the investigated epistemic uncertainties are both the geometric uncertainty of the soil deposit, namely the deposit thickness $(\tilde{h})$, and the material uncertainties such as the shear modulus $\left(\tilde{G}_{0}\right)$, the soil unit density $\rho$, and the damping ratio $\left(\tilde{\xi_{f}}\right)$. The input motion has been modelled as a stochastic stationary Gaussian process. The main goal of this contribution is the formulation of hybrid fuzzy stochastic site response analysis approach; while the seismic input is traditionally considered as random, few contributions investigated the impact of the soil uncertainty on the surface ground motion and, eventually, on the engineering structure. In order to avoid any overestimation effect (see e.g., Guerra et al., [45]), the precise extension principle is applied and a Differential Evolution algorithm (Stefanini 26) has been used to reduce the computational effort of the box-constrained optimization problem. In this paper, the fuzzy output is defined as the median value of the largest peak of the accelerations at the ground surface determined for various $\alpha$-cuts representing the grade of membership to the interval. Results showed a strong influence of the soil parameter uncertainty to the seismic response of the soil deposits, especially for softer types of soil. In ground type D, the dispersion of the median value of the largest peak of the acceleration at the ground surface is higher than the maximum dispersion assumed for the input parameters. Moreover, the results showed that the soil uncertainty can lead to an overestimation or underestimation of the seismic response. A sensitivity analysis showed the importance of the characterization of the shear modulus on the evaluation of the peak ground acceleration. Finally, a gradual elastic response spectrum has been proposed in order to evaluate the impact of the soil uncertainties on the structural design. Especially for softer soils, a structure with a period, $T=0.5 \mathrm{~s}$, resulted strongly affected by the uncertainty of the soil parameters. In particular, it has been shown that a reliable assessment of 
the maximum peak ground acceleration is not a sufficient condition to ensure a safe structural design, while the optimization procedure should be extended to consider the combined effect of soil and structure.

\section{References}

[1] S. L. Kramer, Geotechnical earthquake engineering, Prentice Hall, Upper Saddle River, N.J., 1996.

[2] P. B. Schnabel, J. Lysmer, H. B. Seed, SHAKE, a computer program for earthquake response analysis on horizontally layered sites, Tech. Rep. 7212, Berkeley : College of Engineering, University of California (1972).

[3] J. Bardet, K. Ichii, C. Lin, EERA - a computer program for equivalentlinear earthquake site response analyses of layered soil deposits (2000).

[4] H. B. Seed, I. M. Idriss, Influence of soil conditions on ground motions during earthquakes, Journal of Soil Mechanics and Foundations Division, ASCE 95 (1) (1969) pp. 99-138.

[5] E. M. Rathje, M. C. Ozbey, Site-specific validation of random vibration theory-based seismic site response analysis, Journal of Geotechnical and Geoenvironmental Engineering 132 (7) (2006) 911-922. doi:10.1061/(ASCE) 1090-0241(2006) 132:7(911).

URL http://ascelibrary.org/doi/abs/10.1061/\%28ASCE\% $291090-0241 \% 282006 \% 29132 \% 3 \mathrm{~A} 7 \% 28911 \% 29$

[6] N. Deng, F. Ostadan, Random vibration theory based seismic site response analysis, in: The 14th World Conference on Earthquake Engineering, Beijing, China, 2008.

[7] G. D. Manolis, Stochastic soil dynamics, Soil Dynamics and Earthquake Engineering 22 (1) (2002) 3-15. doi:10.1016/S0267-7261(01)00055-0. URL http://linkinghub.elsevier.com/retrieve/pii/ S0267726101000550 
[8] J. E. Andrade, R. I. Borja, Quantifying sensitivity of local site response models to statistical variations in soil properties, Acta Geotechnica 1 (1) (2006) 3-14. doi:10.1007/s11440-005-0002-4.

URL http://link.springer.com/10.1007/s11440-005-0002-4

[9] E. M. Rathje, A. R. Kottke, W. L. Trent, Influence of input motion and site property variabilities on seismic site response analysis, Journal of Geotechnical and Geoenvironmental Engineering 136 (4) (2010) 607-619. doi:10.1061/(ASCE)GT.1943-5606.0000255

URL http://ascelibrary.org/doi/abs/10.1061/\%28ASCE\%29GT. 1943-5606.0000255

[10] M. Oberguggenberger, W. Fellin, From probability to fuzzy sets: the struggle for meaning in geotechnical risk assessment, in: Probabilistics in Geotechnics: Technical and Economic Risk Estimation, r. pttler, h. klapperich, h. f. schweiger Edition, Verlag Glckauf GmbH, Essen, 2002, pp. pp. $29-38$.

[11] J. Booker, T. Ross, An evolution of uncertainty assessment and quantification, Scientia Iranica 18 (3) (2011) 669-676. doi:10.1016/j.scient.2011.04.017.

URL http://linkinghub.elsevier.com/retrieve/pii/ S1026309811000551

[12] A. T. Beck, W. J. S. Gomes, F. Bazan, ON THE ROBUSTNESS OF STRUCTURAL RISK OPTIMIZATION WITH RESPECT TO EPISTEMIC UNCERTAINTIES, International Journal for Uncertainty Quantification 2 (1) (2012) 1-20. doi:10.1615/Int.J. UncertaintyQuantification.v2.i1.20.

URL http://www.dl.begellhouse.com/journals/52034eb04b657aea, 69f226067bce0f5b, 48708f7546f 160d5.html

[13] M. Beer, S. Ferson, V. Kreinovich, Imprecise probabilities in engineering analyses, Mechanical Systems and Signal Processing 37 (1-2) (2013) 4-29. 
doi:10.1016/j.ymssp.2013.01.024.

URL http://linkinghub.elsevier.com/retrieve/pii/ S0888327013000812

[14] L. Zadeh, Fuzzy sets, Information and Control 8 (3) (1965) 338-353. doi : 10.1016/S0019-9958(65)90241-X

URL http://linkinghub.elsevier.com/retrieve/pii/ S001999586590241X

[15] British Standards Institution., European Committee for Standardization., British Standards Institution., Standards Policy and Strategy Committee., Eurocode 8, design of structures for earthquake resistance, British Standards Institution, London, 2005.

[16] M. Hanss, Applied fuzzy arithmetic: an introduction with engineering applications, Springer-Verlag, Berlin ; New York, 2005.

[17] B. Bede, Mathematics of Fuzzy Sets and Fuzzy Logic, Vol. 295 of Studies in Fuzziness and Soft Computing, Springer Berlin Heidelberg, Berlin, Heidelberg, 2013, dOI: 10.1007/978-3-642-35221-8.

URL http: //link . springer . com/10.1007/978-3-642-35221-8

[18] T. J. Ross, Fuzzy logic with engineering applications, fourth edition Edition, Wiley, Southern Gate, Chichester, West Sussex, United Kingdom, 2017.

[19] Z. Zhang, M. T. Tumay, Statistical to fuzzy approach toward CPT soil classification, Journal of Geotechnical and Geoenvironmental Engineering 125 (3) (1999) 179-186. doi:10.1061/(ASCE) 1090-0241(1999)125: 3(179).

URL http://ascelibrary.org/doi/abs/10.1061/\%28ASCE\% 291090-0241\%281999\%29125\%3A3\%28179\%29

[20] M. Romo, S. Garcia, Neurofuzzy mapping of CPT values into soil dynamic properties, Soil Dynamics and Earthquake Engineering 23 (6) (2003) 
473-482. doi:10.1016/S0267-7261(03)00066-6.

URL http://linkinghub.elsevier.com/retrieve/pii/ S0267726103000666

[21] P. Provenzano, A fuzzy-neural network method for modeling uncertainties in soil-structure interaction problems, Computer-Aided Civil and Infrastructure Engineering 18 (6) (2003) 391-411. doi:10.1111/1467-8667. 00326

URL http://doi.wiley.com/10.1111/1467-8667.00326

[22] M. Valdebenito, C. Prez, H. Jensen, M. Beer, Approximate fuzzy analysis of linear structural systems applying intervening variables, Computers \& Structures 162 116-129. doi:10.1016/j.compstruc.2015.08.020.

URL https://linkinghub.elsevier.com/retrieve/pii/ S004579491500262X

[23] G. C. Marano, E. Morrone, S. Sgobba, S. Chakraborty, A fuzzy random approach of stochastic seismic response spectrum analysis, Engineering Structures 32 (12) (2010) 3879-3887. doi:10.1016/j.engstruct.2010.09.001 URL http://linkinghub.elsevier.com/retrieve/pii/ S0141029610003378

[24] R. W. Clough, Dynamics of structures, McGraw-Hill, New York, 1975.

[25] A. de Luca di Roseto, A. Palmeri, A. G. Gibb, Performance-based seismic design of steel structures accounting for fuzziness in their joint flexibility, Soil Dynamics and Earthquake Engineering 115 799-814. doi:10.1016/j.soildyn.2018.09.007.

URL https://linkinghub.elsevier.com/retrieve/pii/ S0267726118303695

[26] L. Stefanini, A differential evolution algorithm for fuzzy extension of functions, in: P. Melin, O. Castillo, E. G. Ramrez, J. Kacprzyk, W. Pedrycz (Eds.), Analysis and Design of Intelligent Systems using Soft Computing 
Techniques, Vol. 41, Springer Berlin Heidelberg, Berlin, Heidelberg, 2007, pp. $377-386$.

URL http://link.springer.com/10.1007/978-3-540-72432-2_38

[27] A. Der Kiureghian, Structural response to stationary excitation, Journal of the Engineering Mechanics Division 106 (6) (1980) 1195-1213.

[28] E. H. Vanmarcke, Properties of spectral moments with applications to random vibration, Journal of the Engineering Mechanics Division 98 (2) (1972) pp. $425-446$.

[29] G. C. Marano, G. Acciani, A. Fiore, A. Abrescia, Integration algorithm for covariance nonstationary dynamic analysis of SDOF systems using equivalent stochastic linearization, International Journal of Structural Stability and Dynamics 15 (2) 1450044. doi:10.1142/S0219455414500448.

URL http://www.worldscientific.com/doi/abs/10.1142/ S0219455414500448

[30] M. Hanss, S. Turrin, A fuzzy-based approach to comprehensive modeling and analysis of systems with epistemic uncertainties, Structural Safety 32 (6) (2010) 433-441. doi:10.1016/j.strusafe.2010.06.003

URL http://linkinghub.elsevier.com/retrieve/pii/ S0167473010000743

[31] T. Fetz, M. Oberguggenberger, J. Jager, D. Koll, G. Krenn, H. Lessmann, R. F. Stark, Fuzzy Models in Geotechnical Engineering and Construction Management, Computer-Aided Civil and Infrastructure Engineering 14 (2) (1999) 93-106. doi:10.1111/0885-9507.00133. URL http://doi.wiley.com/10.1111/0885-9507.00133

[32] L. Stefanini, L. Sorini, M. L. Guerra, Fuzzy Numbers and Fuzzy Arithmetic in: W. Pedrycz, A. Skowron, V. Kreinovich (Eds.), Handbook of Granular Computing, John Wiley \& Sons, Ltd, Chichester, UK, 2008, pp. 249-283, dOI: 10.1002/9780470724163.ch12.

URL http://doi .wiley.com/10.1002/9780470724163.ch12 
[33] R. J. Aumann, Integrals of set-valued functions Journal of Mathematical Analysis and Applications 12 (1) (1965) 1-12. doi:10.1016/0022-247X(65)90049-1.

URL http://linkinghub.elsevier.com/retrieve/pii/ $0022247 \times 65900491$

[34] T. D. Pham, S. Valliappan, Constructing the membership function of a fuzzy set with objective and subjective information, Computer-Aided Civil and Infrastructure Engineering 8 (1) (1993) 75-82. doi:10.1111/j. 1467-8667.1993.tb00194.x. URL http://doi.wiley.com/10.1111/j.1467-8667.1993.tb00194.x

[35] L. Stefanini, M. L. Guerra, On possibilistic representations of fuzzy intervals, Information Sciences 405 (2017) 33-54. doi: $10.1016 / j$. ins.2017.04.004

URL http://linkinghub.elsevier.com/retrieve/pii/ S002002551730645X

[36] D. Dubois, H. M. Prade, Fuzzy sets and systems: theory and applications, no. v. 144 in Mathematics in science and engineering, Academic Press, New York, 1980.

[37] J. Fortin, D. Dubois, H. Fargier, Gradual numbers and their application to fuzzy interval analysis $16 \quad$ (2) (2008) 388-402. doi:10.1109/TFUZZ.2006.890680.

URL http://ieeexplore.ieee.org/lpdocs/epic03/wrapper.htm? arnumber $=4358793$

[38] P. Cacciola, P. Colajanni, G. Muscolino, Combination of modal responses consistent with seismic input representation, Journal of Structural Engineering 130 (1) (2004) 47-55. doi:10.1061/(ASCE) 0733-9445(2004)130: 1(47)

URL http://ascelibrary.org/doi/abs/10.1061/\%28ASCE\% $290733-9445 \% 282004 \% 29130 \% 3 A 1 \% 2847 \% 29$ 
[39] H. B. Seed, J. I. Sun, Implications of site effects in the mexico city earthquake of sept. 19, 1985 for earthquake-resistant design criteria in the san francisco bay area of california, Tech. Rep. UCB/EERC-89/03, Earthquake Engineering Research Center,, University of California, Berkeley (1989).

[40] I. M. Idriss, Response of soft soil sites during earthquakes, in: Memorial Symposium to honor Professor Harry Bolton Seed, Vol. II, Berkeley, California, 1990.

[41] R. Greco, A. Fiore, G. C. Marano, B. Briseghella, Effects of excitation bandwidth on damping reduction factor, Journal of Earthquake Engineering 1-2\&doi:10.1080/13632469.2018.1528910.

URL https://www.tandfonline.com/doi/full/10.1080/13632469. 2018.1528910

[42] A. Tombari, M. H. El Naggar, F. Dezi, Impact of ground motion duration and soil non-linearity on the seismic performance of single piles, Soil Dynamics and Earthquake Engineering 100 72-87. doi:10.1016/j.soildyn.2017.05.022.

URL http://linkinghub.elsevier.com/retrieve/pii/ S0267726116306765

[43] R. Greco, I. Vanzi, D. Lavorato, B. Briseghella, Seismic duration effect on damping reduction factor using random vibration theory, Engineering Structures 179 296-309. doi:10.1016/j.engstruct.2018.10.074.

URL https://linkinghub.elsevier.com/retrieve/pii/ S0141029618313907

[44] M. Gotz, W. Graf, M. Kaliske, Enhanced uncertain structural analysis with time- and spatial-dependent (functional) fuzzy results, Mechanical Systems and Signal Processing 119 23-38. doi:10.1016/j.ymssp.2018.08.041.

URL

https://linkinghub.elsevier.com/retrieve/pii/ S0888327018305806 
[45] M. L. Guerra, L. Sorini, L. Stefanini, Value function computation in fuzzy models by differential evolution 19 (4) 1025-1031. doi:10.1007/ s40815-017-0308-z.

URL http://link.springer.com/10.1007/s40815-017-0308-z 